\title{
Highly Stereoselective Synthesis of Trisubstituted vinylcyclopropane Derivatives via Arsonium Ylides
}

\author{
Hong Jiang, Xianming Deng, Xiuli Sun and Yong Tang ${ }^{*}$, Li-Xin Dai \\ State Key Laboratory of Organometallic Chemistry, Shanghai Institute of Organic \\ Chemistry, 354 Fenglin Lu, Shanghai 200032, China. \\ tangy@mail.sioc.ac.cn
}

Supporting Information

\section{Contents}

General Information..................................................................S2

Spectral Data of All New Compounds.............................................S2

Copies of NMR Spectra of All New Compounds....................................S7 
General Information: All reactions were carried out under a nitrogen atmosphere in oven-dried glassware with stirring. Tetrahydrofuran was dried by distillation over sodium/benzophenone ketyl.

\section{Spectral Data of All New Compounds:}

Diethyl trans-2-methyl-3-styrylcyclopropane-1,1-dicarboxylate(3b). ${ }^{1} \mathrm{H} \quad \mathrm{NMR}(300 \mathrm{MHz}$, $\left.\mathrm{CDCl}_{3}\right) \delta$ 7.35-7.15 (m, 5H), $6.63(\mathrm{~d}, J=15.9 \mathrm{~Hz}, 1 \mathrm{H}), 5.87$ (dd, $\left.J=15.9,9.3 \mathrm{~Hz}, 1 \mathrm{H}\right), 4.35-4.05$ (m, 4H), 2.55 (dd, J = 9.0, 7.5Hz, 1H), 2.25-2.14 (m, 1H), 1.35-1.15 (m, 9H); ${ }^{13} \mathrm{C}$ NMR(300 MHz, $\left.\mathrm{CDCl}_{3}\right) \delta 168.1,167.7,136.8,133.0,128.5,127.3,125.9,124.9,61.4,42.3,36.5,27.3,14.2,14.1$, 12.3; MS (EI) m/z (rel intensity) 302 (M+ 7), 155 (100), 228 (61), 211 (42), 183 (37), 256 (35), 156 (35), 115 (34), 141 (26); IR (thin film)/cm-1: 2981 (m), 1724 (s), 1368 (m), 1290 (m), 1203 (m), 1140 (m), 964 (m), 693 (m); Anal. Calcd for $\mathrm{C}_{18} \mathrm{H}_{22} \mathrm{O}_{4}$ : C, 71.50; H, 7.33. Found: C, 71.83; H, 7.57.

Diethyl trans-2-isopropyl-3-styrylcyclopropane-1,1-dicarboxylate(3c). $\quad{ }^{1} \mathrm{H}$ NMR (300 MHz, $\left.\mathrm{CDCl}_{3}\right) \delta 7.34-7.14(\mathrm{~m}, 5 \mathrm{H}), 6.63(\mathrm{~d}, J=15.9 \mathrm{~Hz}, 1 \mathrm{H}) 5.86(\mathrm{dd}, J=15.9,9 \mathrm{~Hz}, 1 \mathrm{H})$, 4.34-4.06 (m, 4H), 2.62 (t, $J=7.8 \mathrm{~Hz}, 1 \mathrm{H}), 1.95$ (dd, $J=10.5,7.8 \mathrm{~Hz}, 1 \mathrm{H}), 1.40-1.18$ (m, 7H), 1.05 (d, $J=6.6 \mathrm{~Hz}, 3 \mathrm{H}), 0.98$ (d, $J=6.9 \mathrm{~Hz}, 3 \mathrm{H}) ;{ }^{13} \mathrm{C} \operatorname{NMR}\left(300 \mathrm{MHz}, \mathrm{CDCl}_{3}\right) \delta 168.1,168.0,136.8$, 132.7, 128.5, 127.3, 126.0, 125.1, 61.4, 42.5, 40.7, 35.3, 27.8, 22.1, 21.5, 14.2, 14.1; MS (EI) m/z (rel intensity) 330 (M+1, 9), 195 (100), 141 (78), 241 (73), 169 (60), 115 (58), 91 (51), 128 (47), 183 (47); IR (thin film)/cm': 2961 (m), 2935 (m), 1723 (s), 1367 (m), 1287 (m), 1200 (m), 1181 (m), 963 (m), 693 (m); Anal. Calcd for $\mathrm{C}_{20} \mathrm{H}_{26} \mathrm{O}_{4}$ : C, 72.70; H, 7.93. Found: C, 73.07; H, 8.13.

Dimethyl trans-2-isobutyl-3-styrylcyclopropane-1,1-dicarboxylate(3d). ${ }^{1} \mathrm{H}$ NMR (300 MHz, $\left.\mathrm{CDCl}_{3}\right) \delta$ 7.40-7.08 (m, 5H), $6.63(\mathrm{~d}, J=15.9 \mathrm{~Hz}, 1 \mathrm{H}), 5.86$ (dd, $\left.J=15.9,9.0 \mathrm{~Hz}, 1 \mathrm{H}\right), 3.76(\mathrm{~s}, 3 \mathrm{H})$, $3.72(\mathrm{~s}, 3 \mathrm{H}), 2.60(\mathrm{t}, J=8.1 \mathrm{~Hz}, 1 \mathrm{H}), 2.22-2.12(\mathrm{~m}, 1 \mathrm{H}), 1.78-1.62(\mathrm{~m}, 1 \mathrm{H}), 1.58-1.40(\mathrm{~m}, 1 \mathrm{H})$, 
1.20-1.06 (m, 1H), 0.94 (t, $J=6.9 \mathrm{~Hz}, 6 \mathrm{H}) ;{ }^{13} \mathrm{C} \mathrm{NMR}\left(300 \mathrm{MHz}, \mathrm{CDCl}_{3}\right) \delta 168.5,168.3,136.8$, 133.1, 128.5, 127.4, 126.0, 124.8, 52.6, 41.4, 36.5, 36.3, 32.0, 28.0, 22.6, 22.1; MS (EI) m/z (rel intensity) 316 (M+1 13), 115 (100), 141 (87), 41 (73), 128 (64), 213 (64), 153 (62), 91 (58), 59 (56); IR (thin film)/cm : 2955 (m), 1728 (s), 1436 (m), 1298 (m), 1213 (m), 1144 (m), 964 (m), 693 (m); HRMS (EI) calcd for $\mathrm{C}_{19} \mathrm{H}_{24} \mathrm{O}_{4}\left[\mathrm{M}^{+}\right]$: 316.1675, found: 316.1665 .

Diethyl trans-2-phenyl-3-styrylcyclopropane-1,1-dicarboxylate (3e) ${ }^{8} .{ }^{1} \mathrm{H}$ NMR (300 MHz, $\left.\mathrm{CDCl}_{3}\right) \delta 7.40-7.19(\mathrm{~m}, 10 \mathrm{H}), 6.78(\mathrm{~d}, J=15.9 \mathrm{~Hz}, 1 \mathrm{H}), 6.04(\mathrm{dd}, J=15.9,8.7 \mathrm{~Hz}, 1 \mathrm{H}), 4.30-4.16$ (m, 2H), 4.00-3.82 (m, 2H), 3.49 (d, $J=7.8 \mathrm{~Hz}, 1 \mathrm{H}), 3.32$ (t, $J=9.0 \mathrm{~Hz}, 1 \mathrm{H}), 1.24$ (t, $J=7.2 \mathrm{~Hz}$, 3H), $0.92(\mathrm{t}, J=7.2 \mathrm{~Hz}, 3 \mathrm{H})$.

Dimethyl trans-2-styryl-3-p-tolylclopropane-1,1-dicarboxylate(3f). Mp 84-85 ${ }^{\circ} \mathrm{C} ;{ }^{1} \mathrm{H}$ NMR (300 MHz, $\left.\mathrm{CDCl}_{3}\right) \delta$ 7.39-7.19 (m, 5H), 7.16-7.06 (m, 4H), 6.78 (d, $\left.J=15.9 \mathrm{~Hz}, 1 \mathrm{H}\right), 6.02$ (dd, $J=$ 15.9, 9.0Hz, 1H), 3.77 (s, 3H), 3.49-3.42 (m, 4H), $3.33(\mathrm{t}, J=8.1 \mathrm{~Hz}, 1 \mathrm{H}), 2.31$ (s, 3H); ${ }^{13} \mathrm{C}$ NMR(300 MHz, $\left.\mathrm{CDCl}_{3}\right) \delta 168.0,166.9,137.1,136.7,133.9,131.1,128.9,128.5,128.3,127.6$, 126.1, 124.0, 52.8, 52.4, 44.4, 36.7, 34.0; MS (EI) m/z (rel intensity) 350 ( $\mathrm{M}^{+}$, 3), 231 (100), 290 (100), 318 (85), 215 (68), 286 (51), 216 (49), 115 (48), 258 (47); IR (thin film)/cm': 2952 (m), 1729 (s), 1519 (m), 1435 (m), 1292 (m), 1211 (m), 1100 (m), 964 (m), 755 (m), 693 (m); HRMS (MALDI/DHB) calcd for $\mathrm{C}_{22} \mathrm{H}_{22} \mathrm{O}_{4} \mathrm{Na}^{+}\left[\mathrm{M}^{+}+\mathrm{Na}\right]$ : 373.1423, Found: 373.1410 .

Diethyl trans-2-(4-bromophenyl)-3-styrylcyclopropane-1,1-dicarboxylate(3g). Mp $71-72{ }^{\circ} \mathrm{C}$; ${ }^{1} \mathrm{H}$ NMR (300 MHz, $\mathrm{CDCl}_{3}$ ) $\delta$ 7.45-7.13 (m, 9H), 6.79 (d, $\left.J=15.9 \mathrm{~Hz}, 1 \mathrm{H}\right), 6.01$ (dd, $J=15.9$, 8.7Hz, 1H), 4.36-4.10 (m, 2H), 4.04-3.84 (m, 2H), $3.42(\mathrm{~d}, J=8.1 \mathrm{~Hz}, 1 \mathrm{H}), 3.28(\mathrm{t}, J=8.1 \mathrm{~Hz}, 1 \mathrm{H})$, 1.25 (t, $J=7.2 \mathrm{~Hz}, 3 \mathrm{H}), 1.00$ (t, $J=6.9 \mathrm{~Hz}, 3 \mathrm{H}) ;{ }^{13} \mathrm{C} \mathrm{NMR}\left(300 \mathrm{MHz}, \mathrm{CDCl}_{3}\right) \delta 167.2,166.1,136.5$, 134.1, 133.5, 131.3, 130.3, 128.5, 127.6, 126.1, 123.6, 121.3, 61.8, 61.5, 44.5, 35.9, 33.5, 14.2, 
13.8; MS (EI) m/z (rel intensity) 443 ( $\mathrm{M}^{+}, 3$ ), 215 (100), 370 (88), 368 (84), 216 (83), 297 (79), 295 (75), 49 (70), 115 (60); IR (thin film)/cm' : 2981 (m), 1724 (s), 1492 (m), 1369 (m), 1289 (m), 1095 (m), 1012 (m), 963 (m), 759 (m), 693 (m); HRMS (MALDI/DHB) calcd for $\mathrm{C}_{23} \mathrm{H}_{23} \mathrm{O}_{4} \mathrm{BrNa}^{+}$ $\left[\mathrm{M}^{+}+\mathrm{Na}\right]:$ 465.0685, Found: 465.0672.

Diethyl trans-2-phenyl-3-vinylcyclopropane-1,1-dicarboxylate (3h) ${ }^{8} .{ }^{1} \mathrm{H}$ NMR $(300 \mathrm{MHz}$, $\left.\mathrm{CDCl}_{3}\right) \delta$ 7.22-7.13 (m, 5H), 5.65-5.10 (m, 1H), $5.36(\mathrm{dd}, \mathrm{J}=17.1,1.5 \mathrm{~Hz}, 1 \mathrm{H}), 5.14$ (dd, $\mathrm{J}=10.2$, 1.8Hz, 1H), 4.28-4.09 (m, 2H), 3.90-3.75 (m, 2H), 3.30 (d, $J=8.1 \mathrm{~Hz}, 1 \mathrm{H}), 3.10$ (t, $J=7.8 \mathrm{~Hz}, 1 \mathrm{H})$, $1.21(\mathrm{t}, J=7.2 \mathrm{~Hz}, 3 \mathrm{H}), 0.84$ (t, $J=7.2 \mathrm{~Hz}, 3 \mathrm{H})$.

Diethyl trans-2-phenyl-3-(2-(trimethylsilyl)vinyl)cyclopropane-1,1-dicarboxylate $\quad(3 \mathbf{j})^{8} \cdot{ }^{1} \mathrm{H}$ NMR (300 MHz, $\left.\mathrm{CDCl}_{3}\right) \delta$ 7.26-7.20 (m, 5H), 6.23 (d, $\left.J=18.3 \mathrm{~Hz}, 1 \mathrm{H}\right), 5.76$ (dd, J = 18.6, 8.1Hz, 1H), 4.24 (q, $J=6.9 \mathrm{~Hz}, 2 \mathrm{H}), 3.95-3.85(\mathrm{~m}, 2 \mathrm{H}), 3.41(\mathrm{~d}, J=7.8 \mathrm{~Hz}, 1 \mathrm{H}), 3.19(\mathrm{t}, J=8.1 \mathrm{~Hz}, 1 \mathrm{H})$, $1.28(\mathrm{t}, J=6.9 \mathrm{~Hz}, 3 \mathrm{H}), 0.91(\mathrm{t}, J=7.2 \mathrm{~Hz}, 3 \mathrm{H}), 0.06(\mathrm{~s}, 9 \mathrm{H})$.

Diethyl trans-2-ethyl-3-(2-(trimethylsilyl)vinyl)cyclopropane-1,1-dicarboxylate (3k). ${ }^{1} \mathrm{H}$ NMR $\left(300 \mathrm{MHz}, \mathrm{CDCl}_{3}\right) \delta 5.93(\mathrm{~d}, J=18.6 \mathrm{~Hz}, 1 \mathrm{H}), 5.57(\mathrm{dd}, \mathrm{J}=18.6,8.1 \mathrm{~Hz}, 1 \mathrm{H}), 4.30-4.08(\mathrm{~m}, 4 \mathrm{H})$, $2.43(\mathrm{t}, J=7.8 \mathrm{~Hz}, 1 \mathrm{H}), 2.04(\mathrm{q}, J=7.8 \mathrm{~Hz}, 1 \mathrm{H}), 1.54-1.30(\mathrm{~m}, 2 \mathrm{H}), 1.23(\mathrm{q}, J=6.9 \mathrm{~Hz}, 6 \mathrm{H}), 0.96$ (t, $J=7.2 \mathrm{~Hz}, 3 \mathrm{H}), 0.00$ (s, 9H); ${ }^{13} \mathrm{C} \operatorname{NMR}\left(300 \mathrm{MHz}, \mathrm{CDCl}_{3}\right) \delta 167.9,140.8,134.2,61.4,61.3$, 42.2, 37.9, 34.1, 20.7, 14.2, 14.1, 13.0, -1.4; MS (EI) m/z (rel intensity) 312 (M+1 4), 75 (100), 73 (91), 223 (52), 283 (49), 59 (37), 121 (31), 91 (27), 205 (24); IR (thin film)/cm-1: 2960 (m), 1726 (s), 1368 (m), 1291 (m), 1249 (m), 1202 (m), 1147 (m), 864 (m), 839 (m); HRMS (EI) calcd for $\mathrm{C}_{16} \mathrm{H}_{28} \mathrm{O}_{4} \mathrm{Si}\left[M^{+}\right]$: 312.1757, found: 312.1774.

Diethyl 2-isopropyl-3-phenylcyclopropane-1,1-dicarboxylate(3m). (trans/cis $=1 / 1$ ) ${ }^{1} \mathrm{H}$ NMR (300 MHz, $\mathrm{CDCl}_{3}$ ) $\delta$ 7.30-7.14 (m, 5H), 4.35-4.15 (m, 2H), 4.15-3.95 (m, 1H), 3.94-3.76 (m, 1H), 
$3.10(\mathrm{~d}, J=8.1 \mathrm{~Hz}, 0.5 \mathrm{H}), 3.06(\mathrm{~d}, J=9.9 \mathrm{~Hz}, 0.5 \mathrm{H}), 2.34-2.14(\mathrm{~m}, 1 \mathrm{H}), 1.67$ (t, $0.5 \mathrm{H}), 1.34-1.24$ (m, 3.5H), 1.16 (dd, $J=9.0,6.6 \mathrm{~Hz}, 3 \mathrm{H}), 1.09$ (t, $J=7.2 \mathrm{~Hz}, 1.5 \mathrm{H}), 1.03$ (d, $J=6.9 \mathrm{~Hz}, 1.5 \mathrm{H}), 0.95$ (d, $J=6.3 \mathrm{~Hz}, 1.5 \mathrm{H}), 0.89$ (t, $J=7.2 \mathrm{~Hz}, 1.5 \mathrm{H})$; MS (EI) $\mathrm{m} / \mathrm{z}$ (rel intensity) $305\left(\mathrm{M}^{+}+1,5\right), 187$ (100), 261 (98), 115 (62), 43 (42), 91 (32), 41 (32), 170 (26), 105 (22); IR (thin film)/cm' : 2978 (m), 2962 (m), 1726 (s), 1368 (m), 698 (m); Anal. Calcd for $\mathrm{C}_{18} \mathrm{H}_{24} \mathrm{O}_{4}$ : C, 71.03; H, 7.95. Found: C, 71.31; H, 8.17.

Dimethyl trans-2-phenyl-3-styrylclopropane-1,1-dicarboxylate(3n). ${ }^{1} \mathrm{H}$ NMR (300 MHz, $\left.\mathrm{CDCl}_{3}\right) \delta 7.40-7.18(\mathrm{~m}, 10 \mathrm{H}), 6.79(\mathrm{~d}, J=15.9 \mathrm{~Hz}, 1 \mathrm{H}), 6.03$ (dd, $\left.J=15.6,8.4 \mathrm{~Hz}, 1 \mathrm{H}\right), 3.78(\mathrm{~s}$, 3H), 3.50 (d, $J=8.4 \mathrm{~Hz}, 1 \mathrm{H}), 3.44$ (s, 3H), 3.34 (t, $J=8.4 \mathrm{~Hz}, 1 \mathrm{H}) ;{ }^{13} \mathrm{C}$ NMR(300 MHz, $\mathrm{CDCl}_{3}$ ) $\delta 167.9,166.8,136.7,134.3,134.1,128.6,128.4,128.2,127.6,127.4,126.2,123.9,52.9,52.4,44$. 5, 36.9, 33.9; MS (EI) m/z (rel intensity) 336 (M+1, 2), 276 (100), 217 (80), 304 (64), 115 (40), 244 (33), 202 (32), 215 (32), 59 (30); IR (thin film)/cm'-1728 (s), 1435 (m), 1292 (m), 1105 (m), 761 (m), 742 (m), 695 (s); Anal. Calcd for $\mathrm{C}_{21} \mathrm{H}_{20} \mathrm{O}_{4}$ : C, 74.98; H, 5.99. Found: C, 74.92; H, 6.09.

Diethyl trans-2,3-diphenylcyclopropane-1,1-dicarboxylate (3o) ${ }^{8} .{ }^{1} \mathrm{H}$ NMR (300 $\left.\mathrm{MHz}, \mathrm{CDCl}_{3}\right)$ $\delta$ 7.40-7.20 (m, 10H), 3.95 (q, $J=7.2 \mathrm{~Hz}, 4 \mathrm{H}), 3.83(\mathrm{~s}, 2 \mathrm{H}), 0.95$ (t, $J=6.9 \mathrm{~Hz}, 6 \mathrm{H})$.

Cinnamyltriphenylarsonium tetraphenylborate(Table 1, entry 4). The salt (5) was obtained by mixing Cinnamytriphenylarsonium bromide $(0.85 \mathrm{~g}, 1.69 \mathrm{mmol})$ with sodium tetraphenylborate (0.65 g, 1.9 mmol) in methanol (20 mL). Yield: 1.16 g (92\%); Mp 160-161 ${ }^{\circ} \mathrm{C} ;{ }^{1} \mathrm{H}$ NMR (300 MHz, $\left.\mathrm{CDCl}_{3}\right) \delta$ 7.64-7.54 (m, 3H), 7.47-7.36 (m, 14H), 7.30-7.20 (m, 3H), 7.14-7.04 (m, 8H), 6.93-6.83 (m, 8H), 6.79-6.70 (m, 4H), 6.14 (d, $J=15.9 \mathrm{~Hz}, 1 \mathrm{H}), 5.64-5.48(\mathrm{~m}, 1 \mathrm{H}), 2.79(\mathrm{~d}, J=$ 7.8Hz, 2H); ${ }^{13} \mathrm{C} \mathrm{NMR}\left(300 \mathrm{MHz}, \mathrm{CDCl}_{3}\right) \delta 165.1,164.4,163.7,163.1,139.5,136.2,135.0,134.3$, 132.3, 128.7, 126.4, 125.6, 125.5, 121.7, 120.0, 113.9, 28.7; MS (ESI, positive mode, m/z) 423 
$\left(\mathrm{M}^{+}-\mathrm{BPh}_{4}\right)$; IR (thin film)/cm'-1 3054 (s), 2998 (m), 1579 (m), 1482 (m), 1439 (m), 1084 (m), 734 (s), 706 (s), 689 (s), 612 (m), 466 (m); Anal. Calcd for $\mathrm{C}_{51} \mathrm{H}_{44}$ AsB: C, 82.48; H, 5.97. Found: C, 82.47; H, 6.00 .

Cinnamyltriphenylarsonium tetrafluoroborate(Table 1, entry 5). A solution of

Cinnamyltriphenyl- arsonium bromide $(1.52 \mathrm{~g}, 3.02 \mathrm{mmol})$ in $\mathrm{CH}_{2} \mathrm{Cl}_{2}(40 \mathrm{~mL})$ is added a solution of silver tetrafluoroborate (680mg, $3.50 \mathrm{mmol})$ in water $(20 \mathrm{~mL})$, and the mixture is stirred vigorously for $10 \mathrm{~min}$. The silver bromide formed is filtered off, the filtrate is allowed to settle and the organic phase is washed with saturated brine $(5 \mathrm{~mL})$ and evaporated to dryness. Yield: 1.51g(97\%); $\quad \mathrm{Mp} 147-148{ }^{\circ} \mathrm{C} ;{ }^{1} \mathrm{H}$ NMR (300 MHz, $\left.\mathrm{CDCl}_{3}\right) \delta$ 7.76-7.60 (m, 15H), 7.35-7.15 (m, 5H), 6.73 (d, $J=15.9 \mathrm{~Hz}, 1 \mathrm{H}), 6.17-6.03(\mathrm{~m}, 1 \mathrm{H}), 4.59$ (d, $J=7.8 \mathrm{~Hz}, 2 \mathrm{H}) ;{ }^{13} \mathrm{C}$ NMR(300 MHz, $\left.\mathrm{CDCl}_{3}\right) \delta 139.8,135.4,134.1,132.8,130.8,128.6,128.4,126.5,120.8,114.5,29.8 ;$ MS (ESI, positive mode, m/z) 423( $\left.\mathrm{M}^{+}-\mathrm{BF}_{4}\right)$; IR (thin film)/ $/ \mathrm{cm}^{-1}$ : 1485 (m), 1440 (s), 1059 (s), 997 (m), 741 (s), 690 (s), 467 (m); HRMS (ESI) calcd for $\mathrm{C}_{27} \mathrm{H}_{24} \mathrm{As}^{+}\left(\mathrm{M}^{+}\right)$: 423.1087, Found: 423.1088. 
2. Copies of NMR Spectra of All New Compounds 

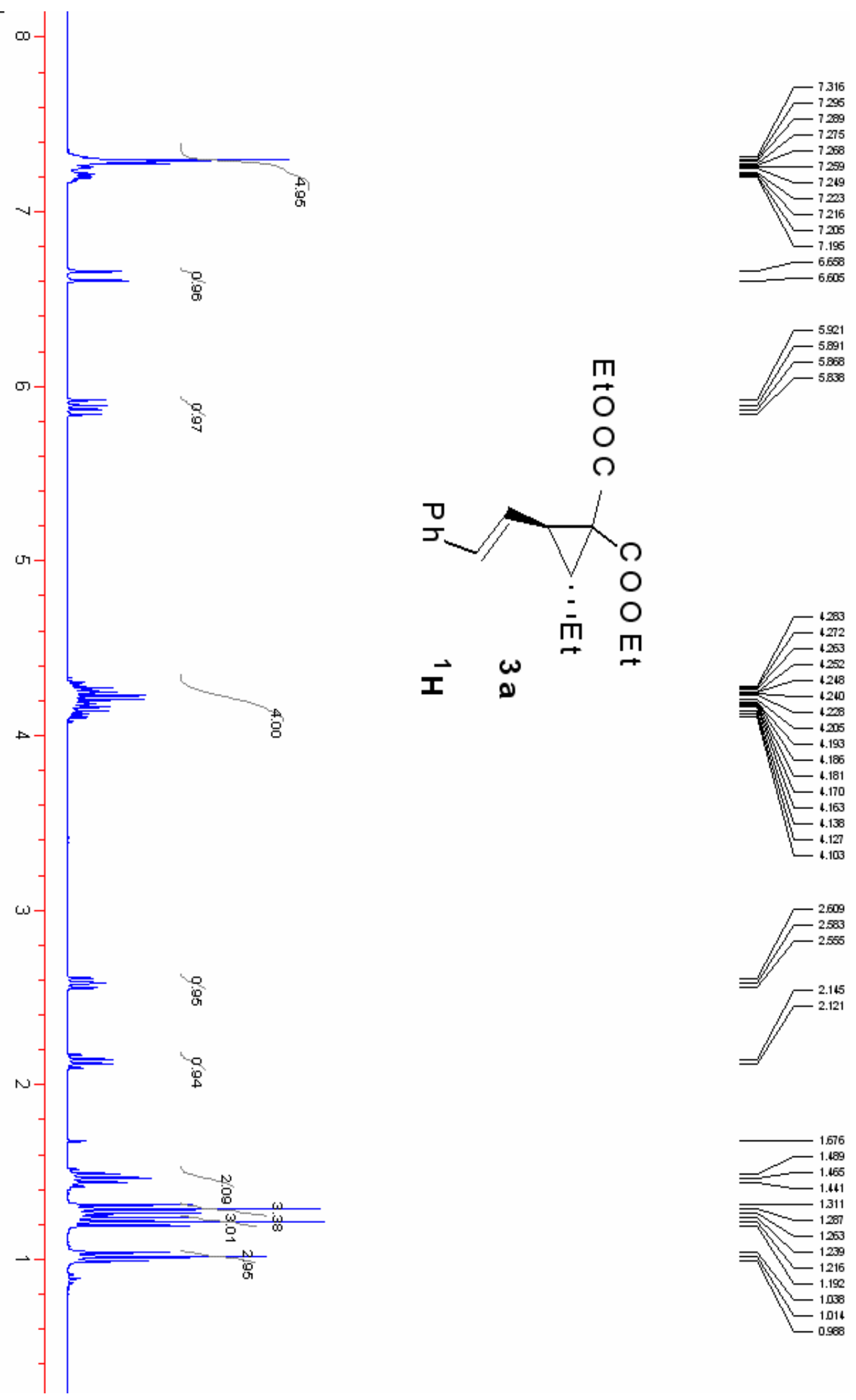


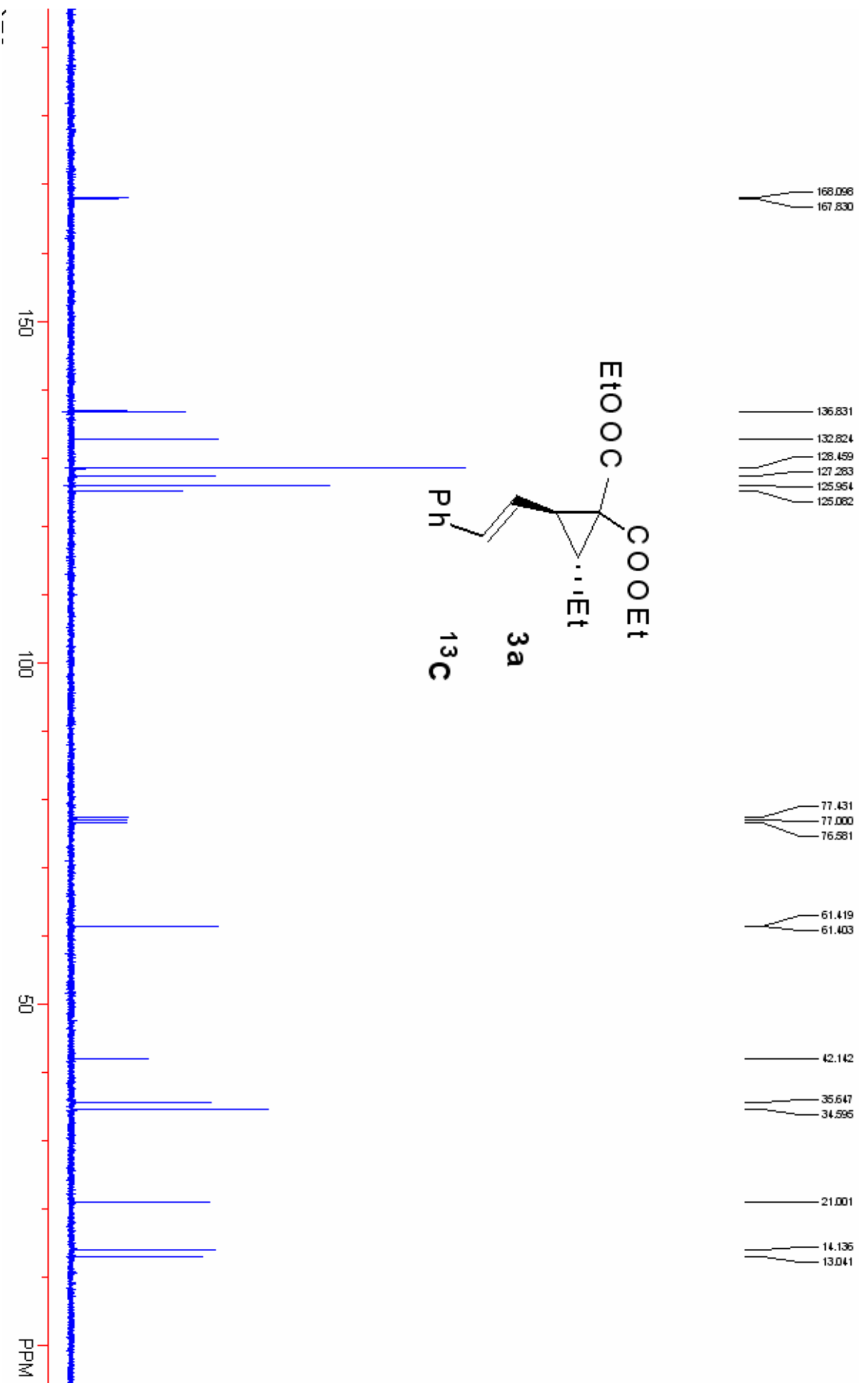



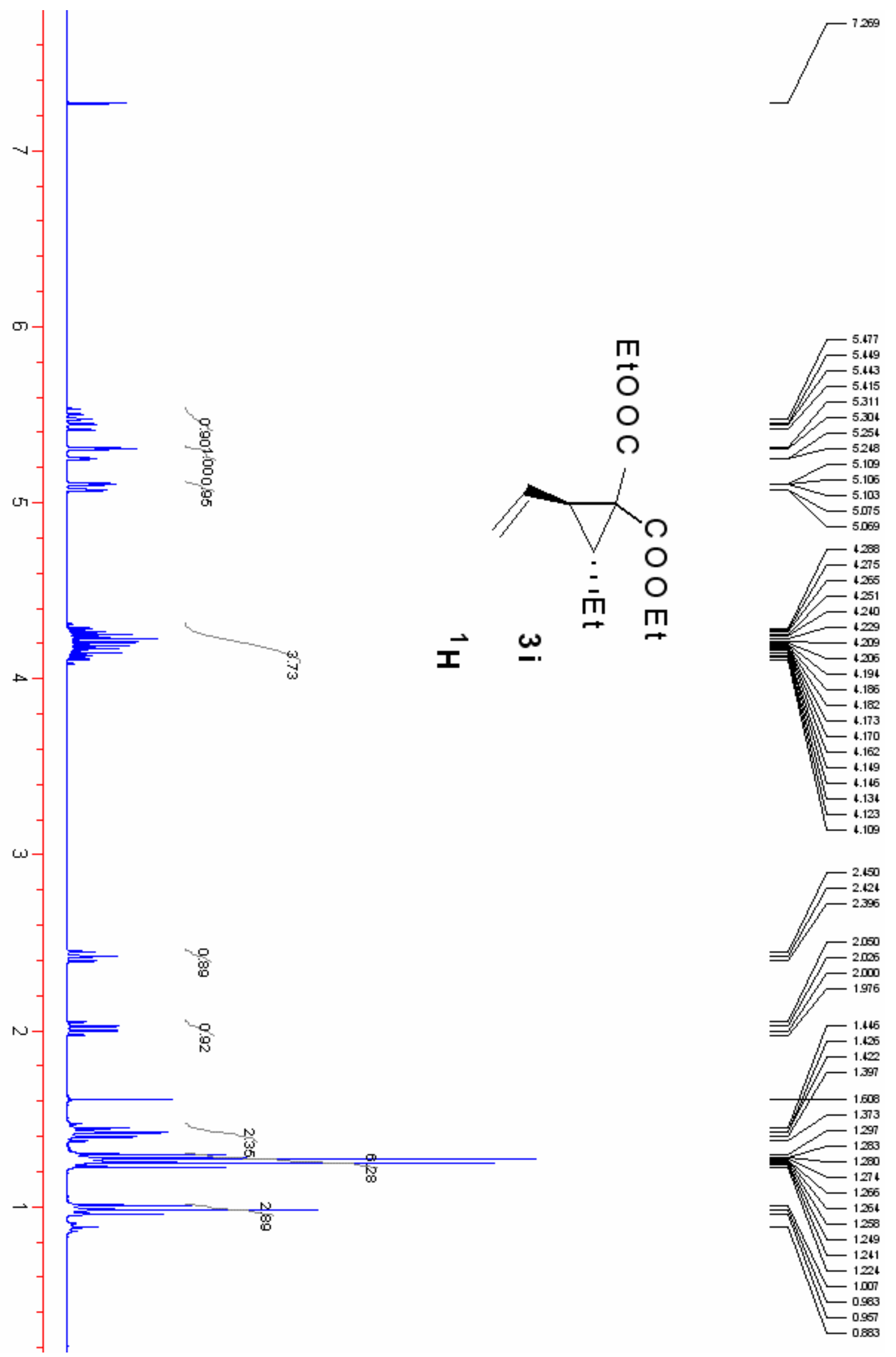

5.109
-5.105
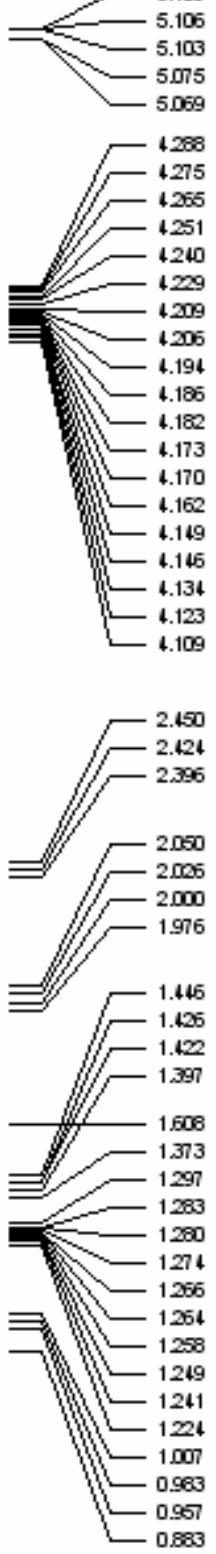

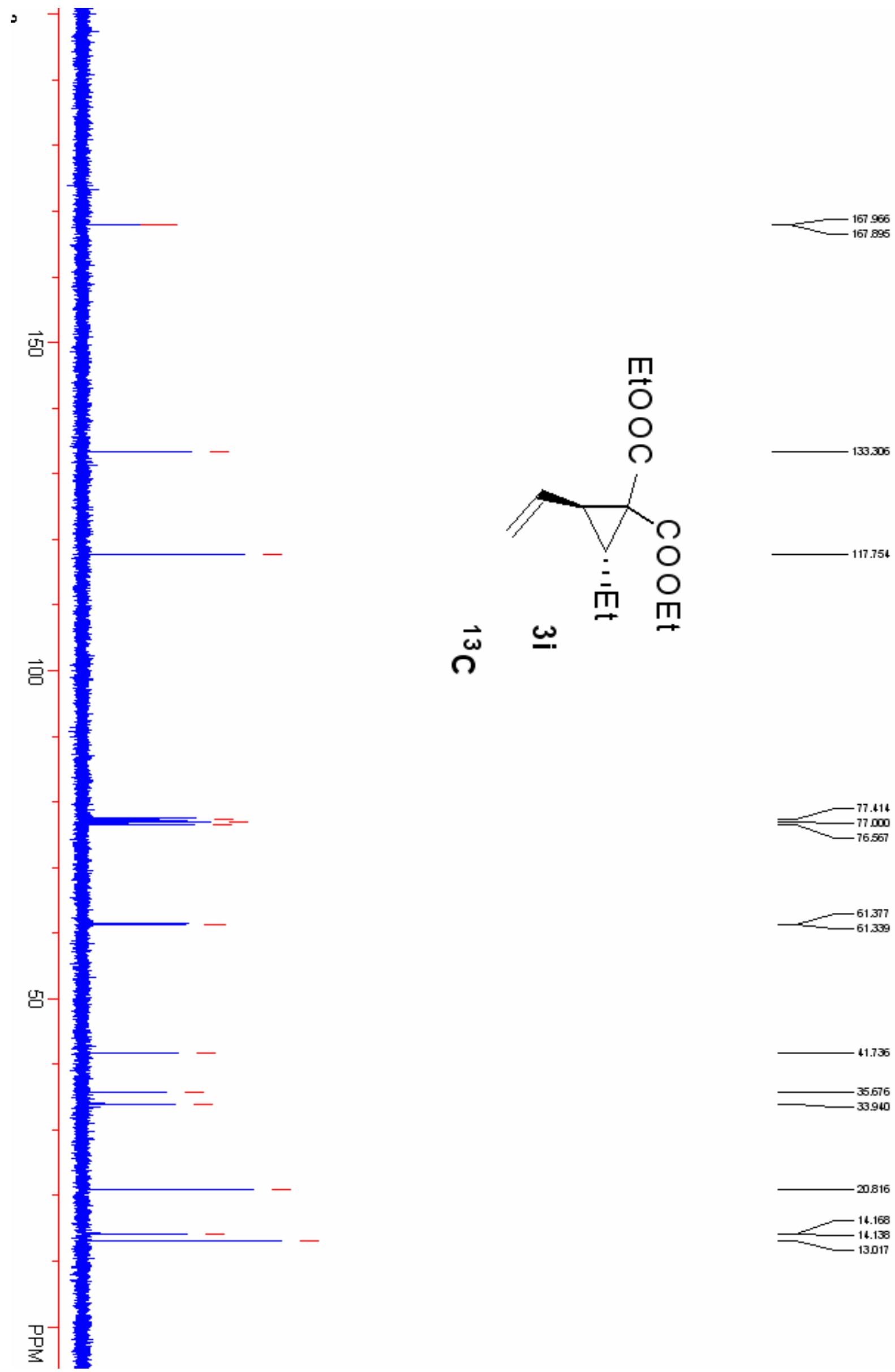

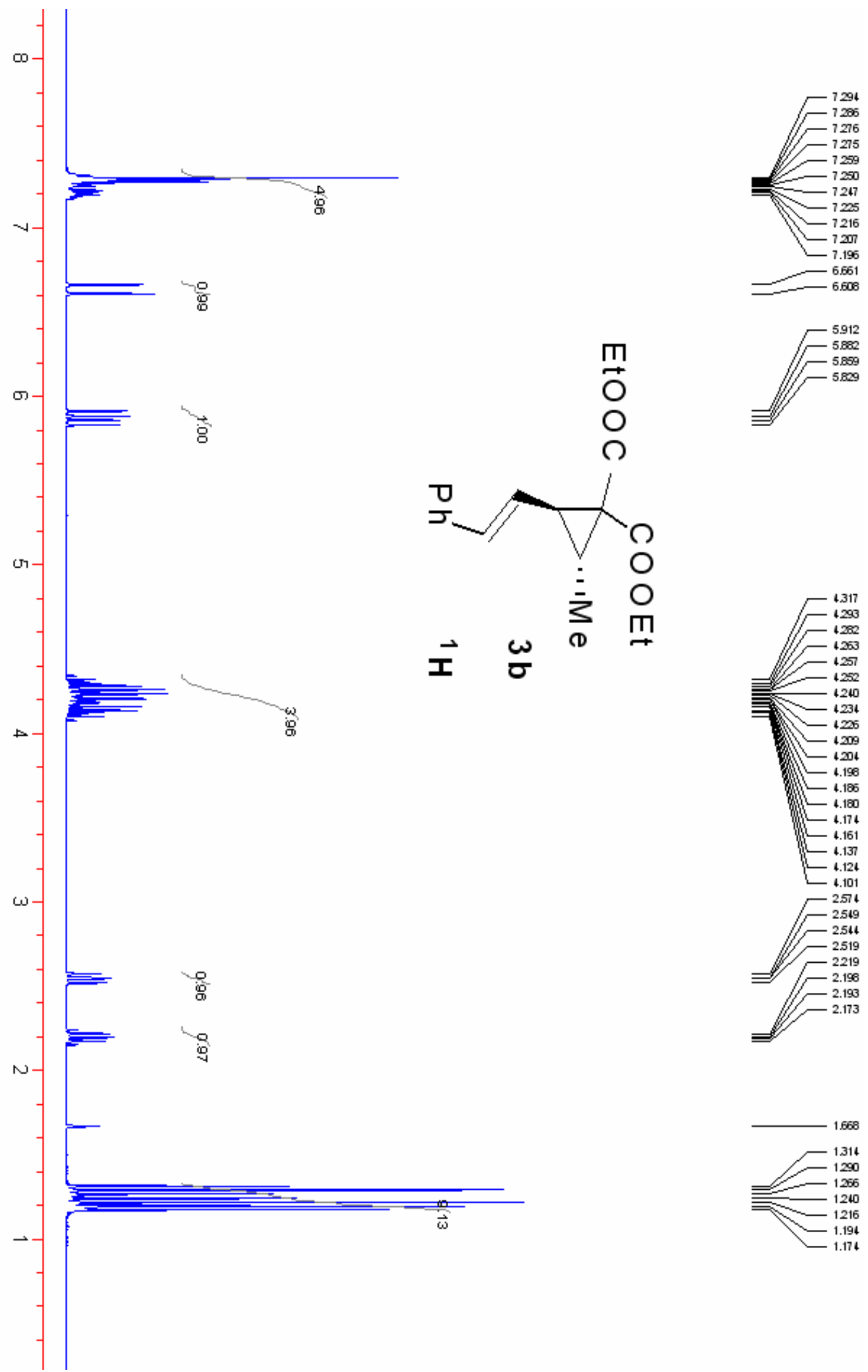


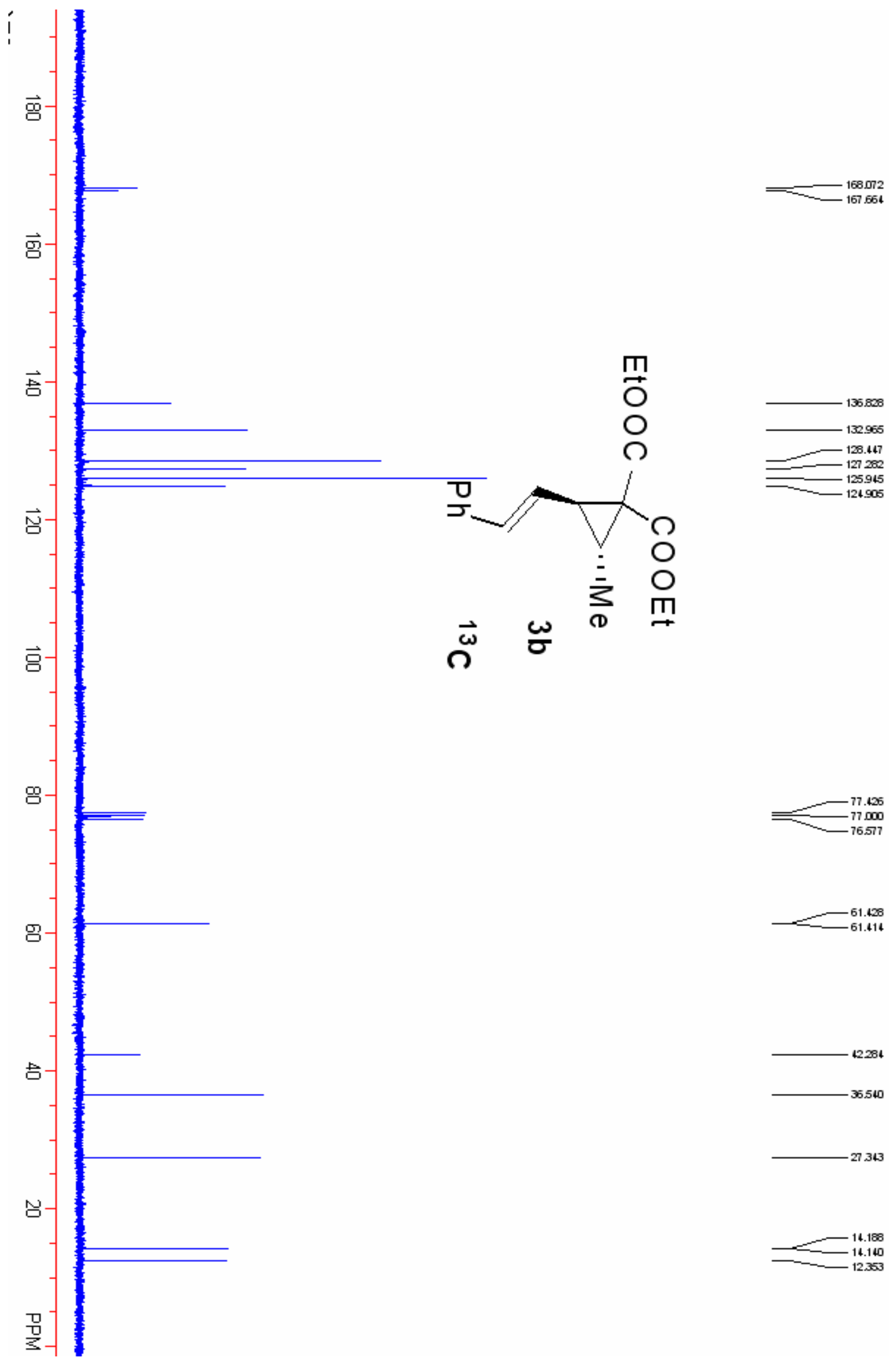



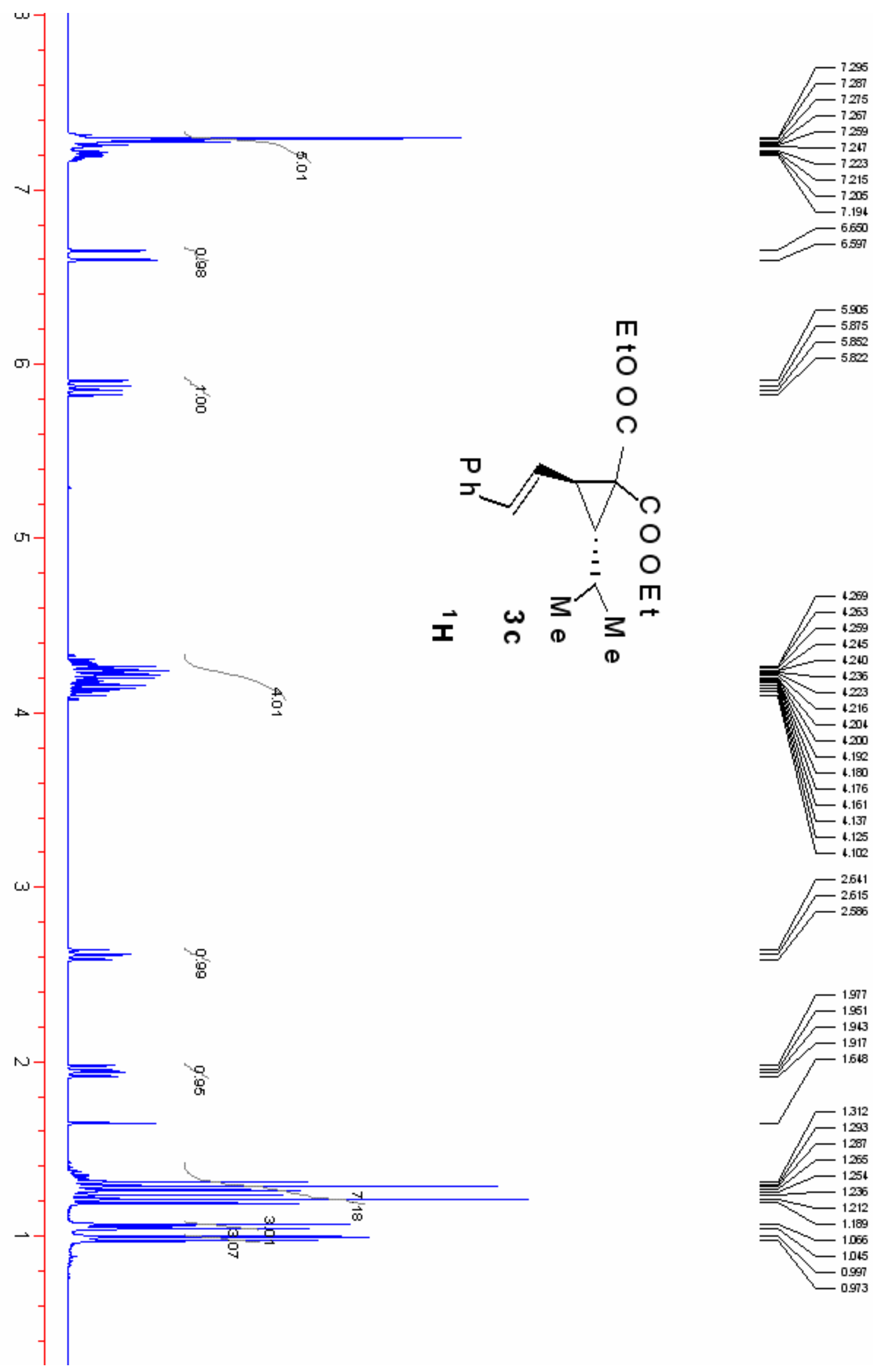

0 

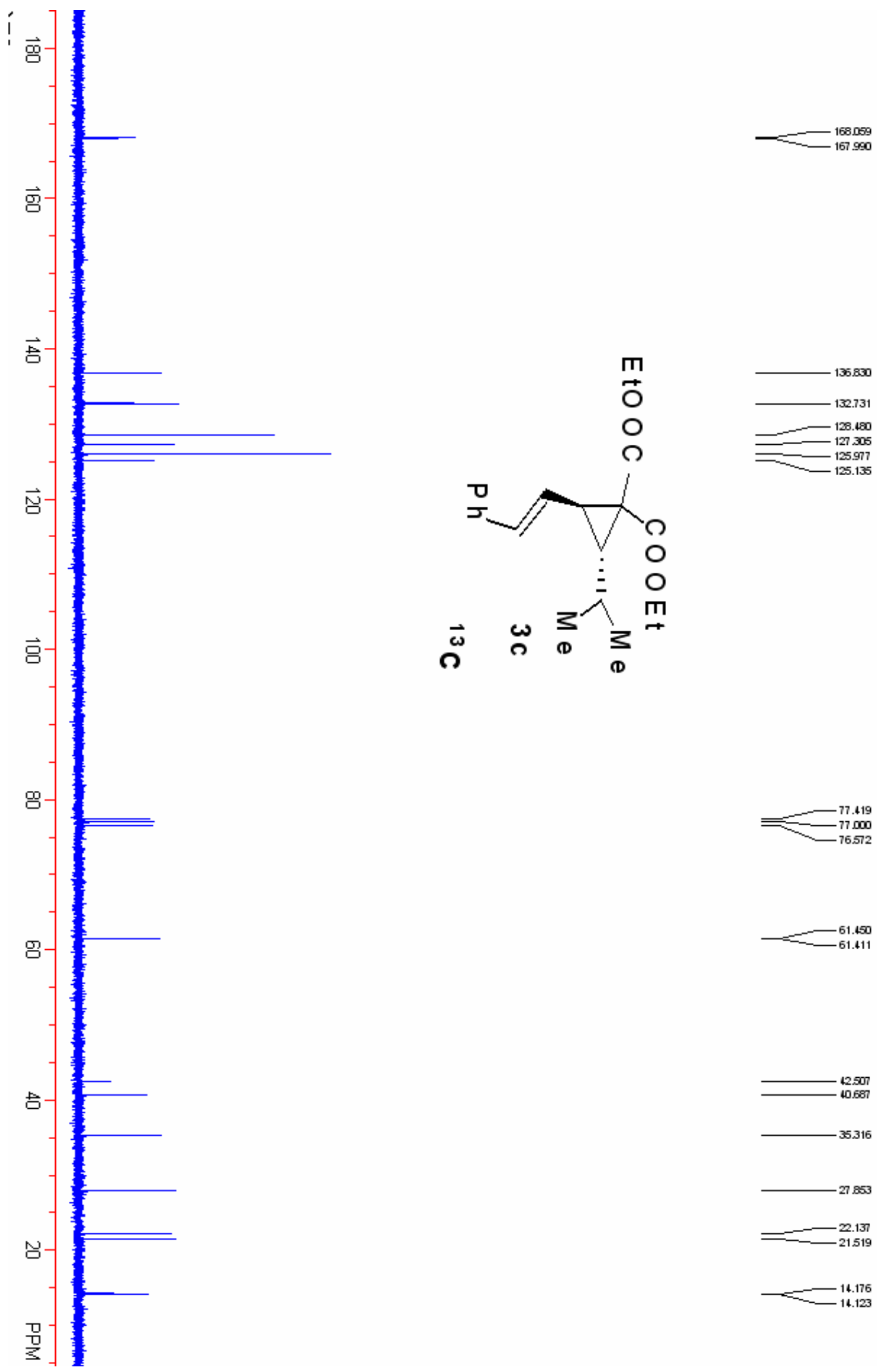

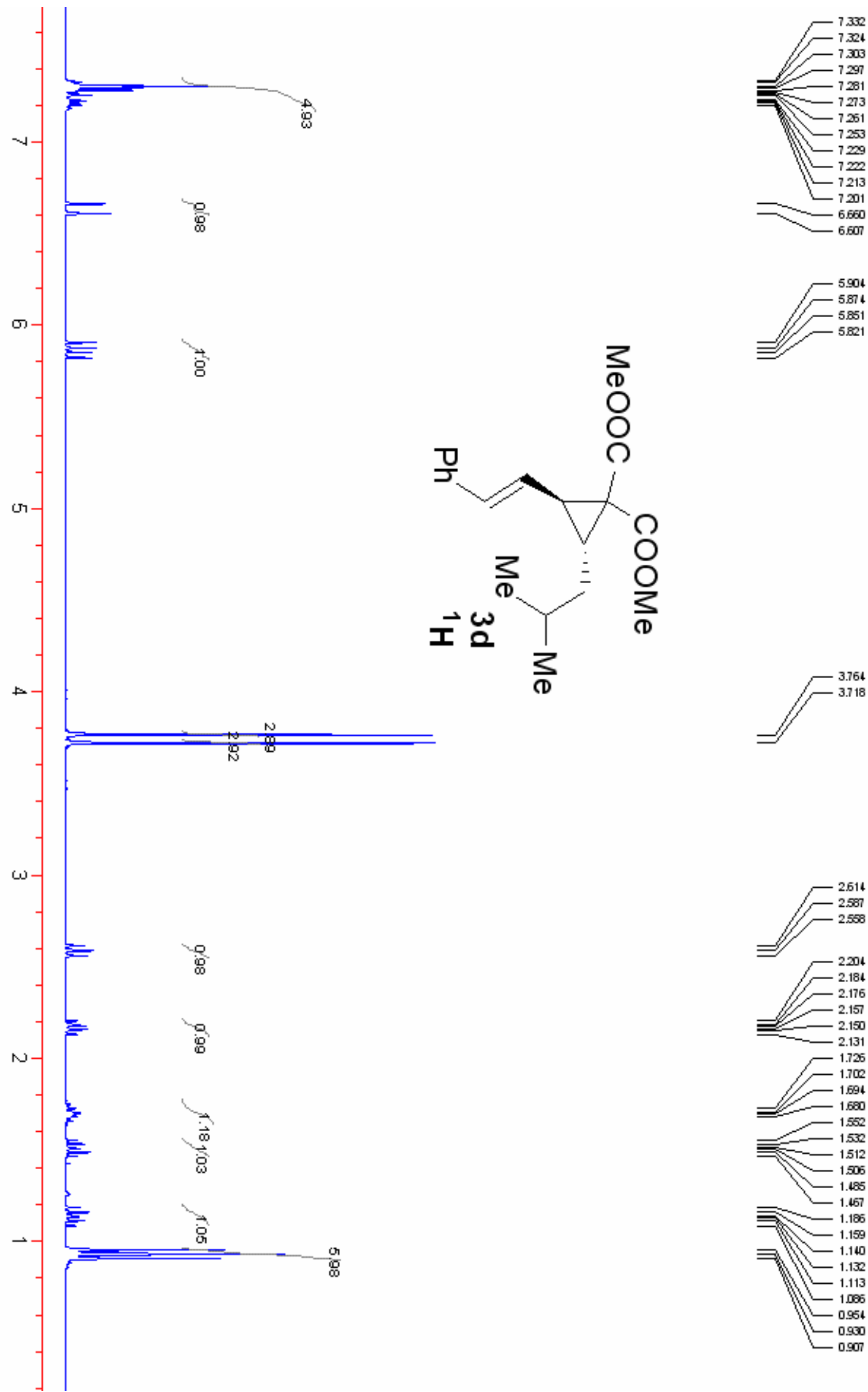

$\frac{1}{10}$

응

믕

工盛
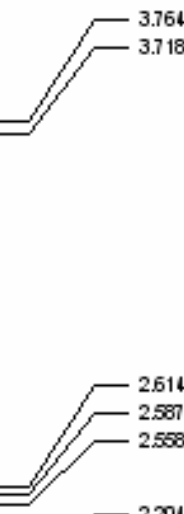

$-220$

2.184

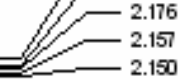

- 1.725

1.702
1.694

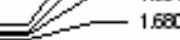

1.552

1.512

$\checkmark-1.506$

三 -1.167

$\Rightarrow-1.16$

11.132

$M=1.113$

- 0.964

0.930

$-0.907$ 


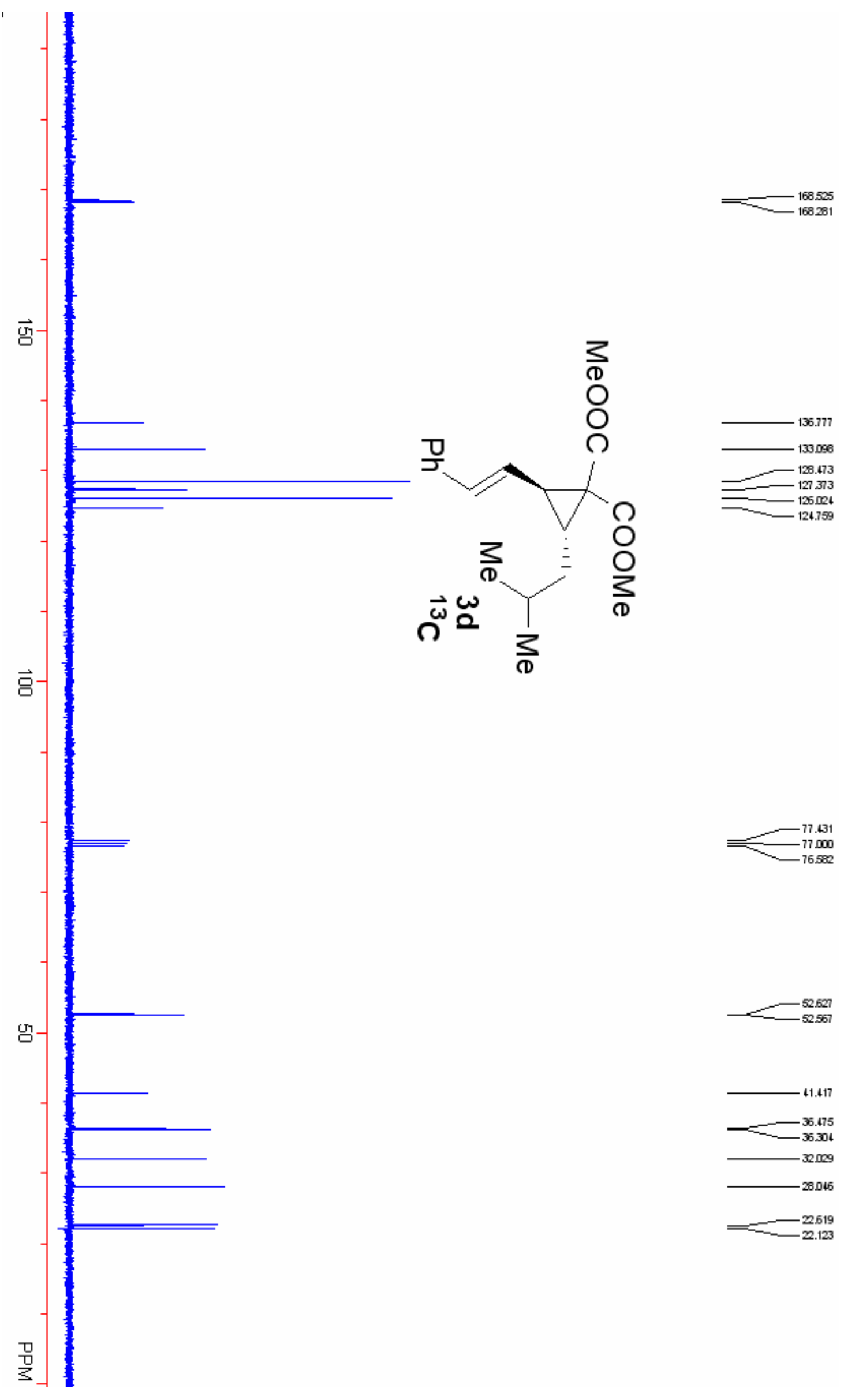




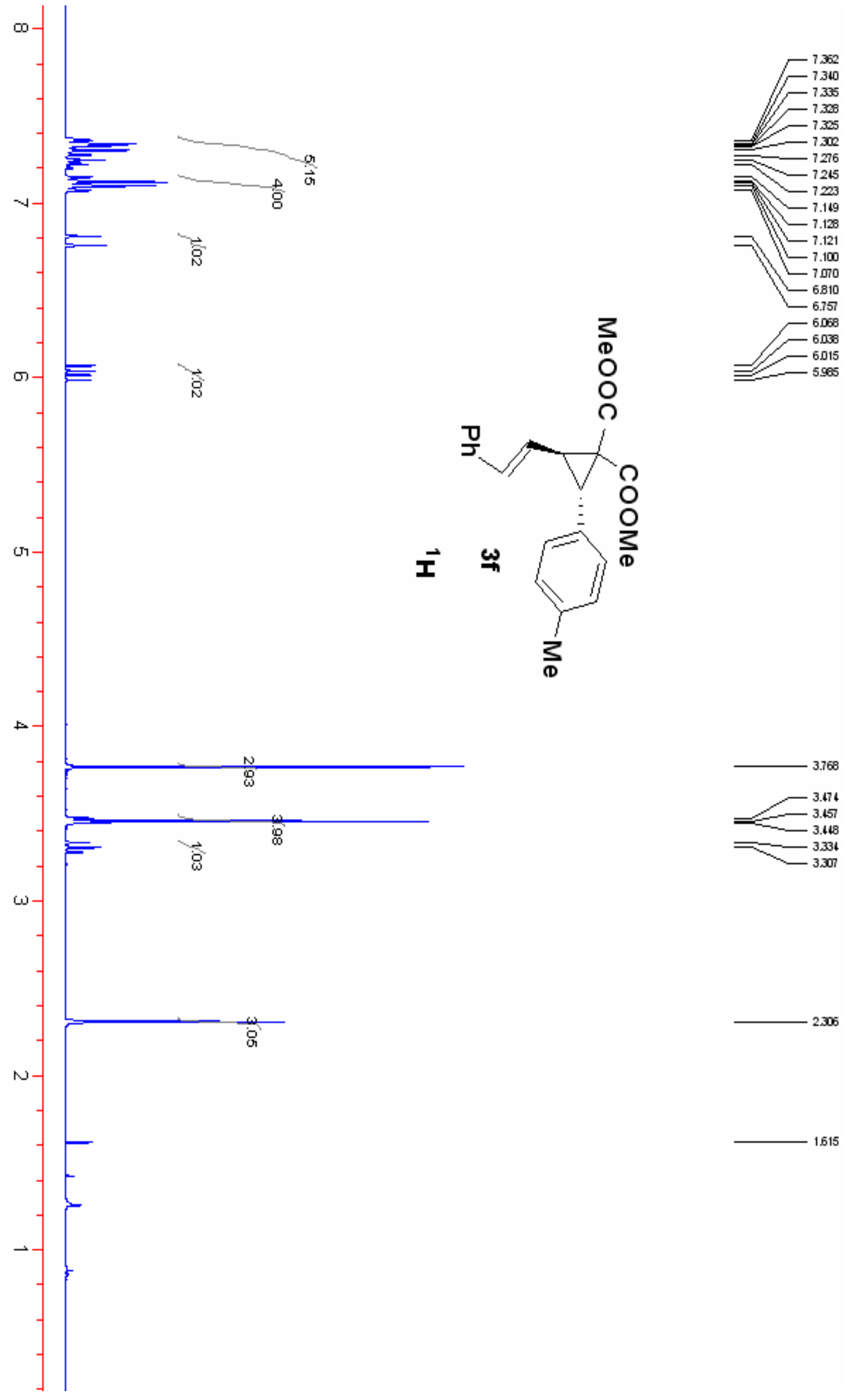



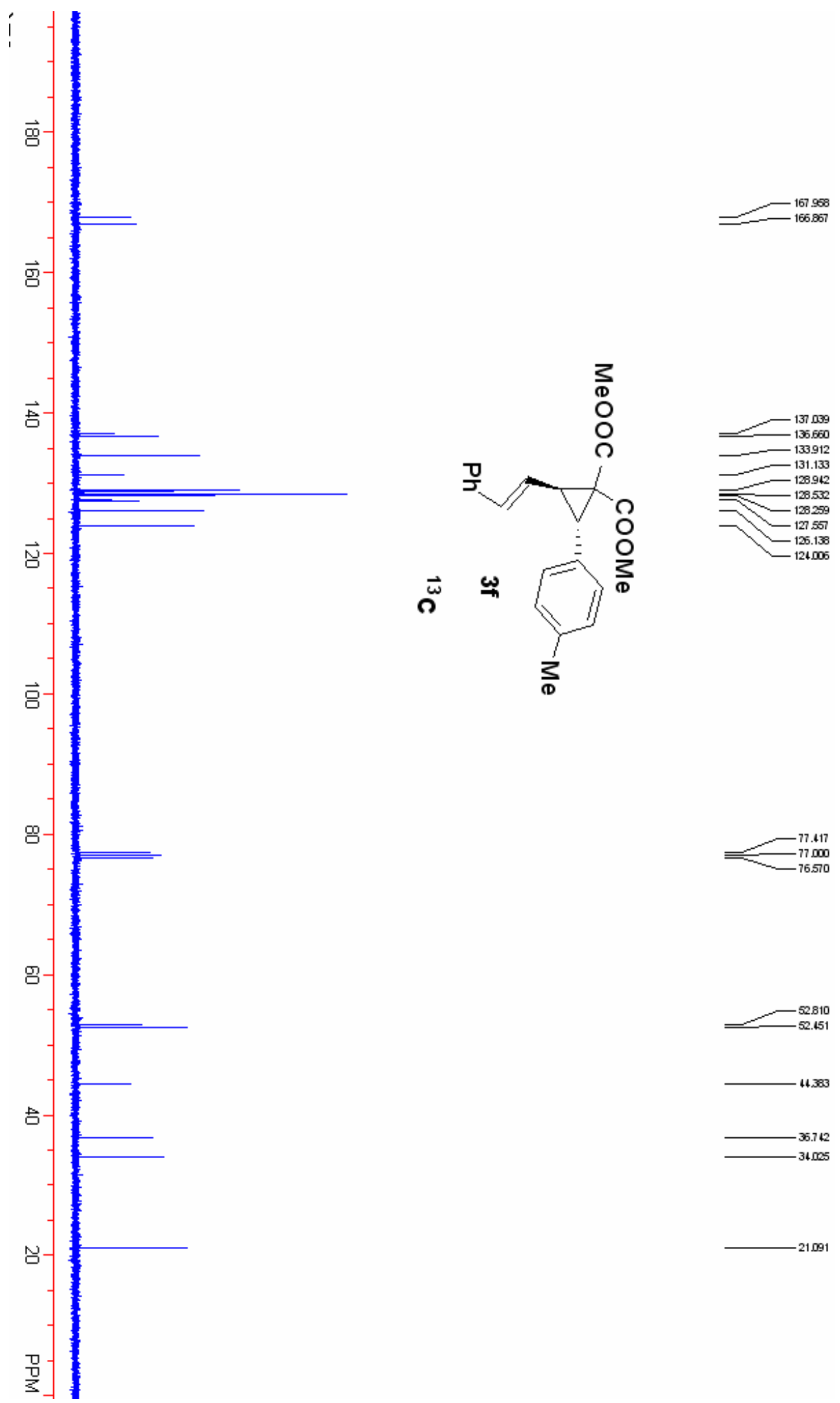

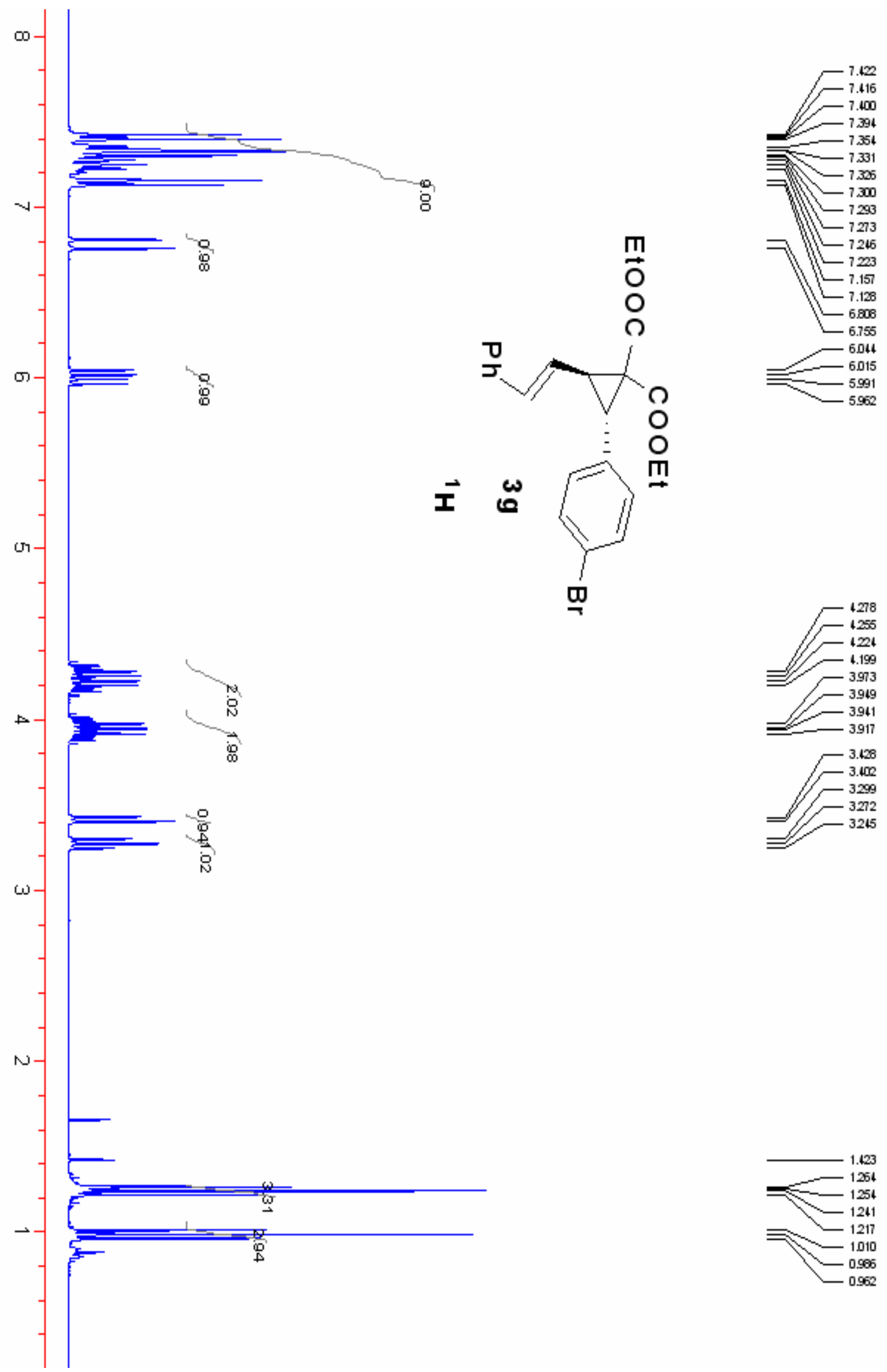


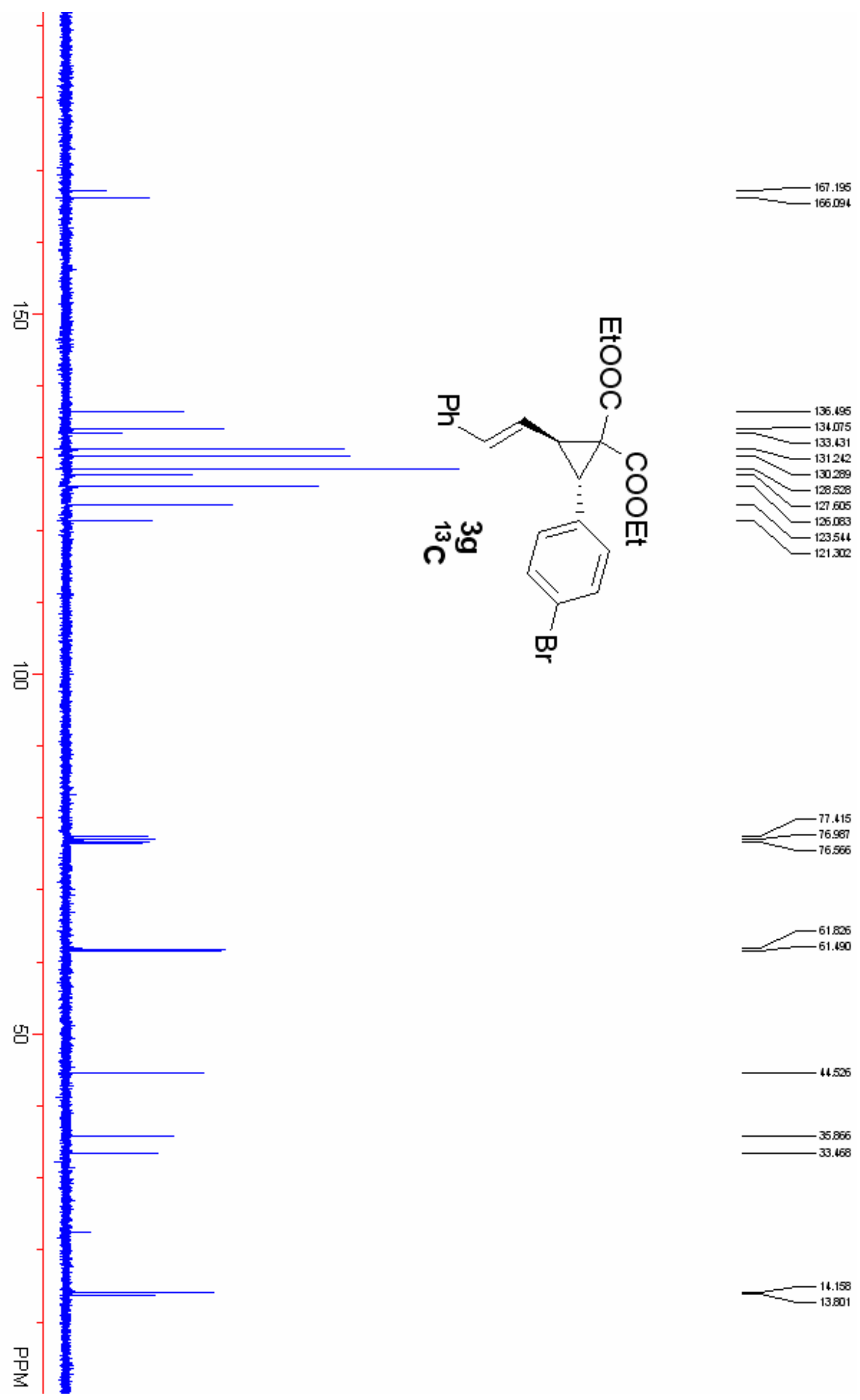



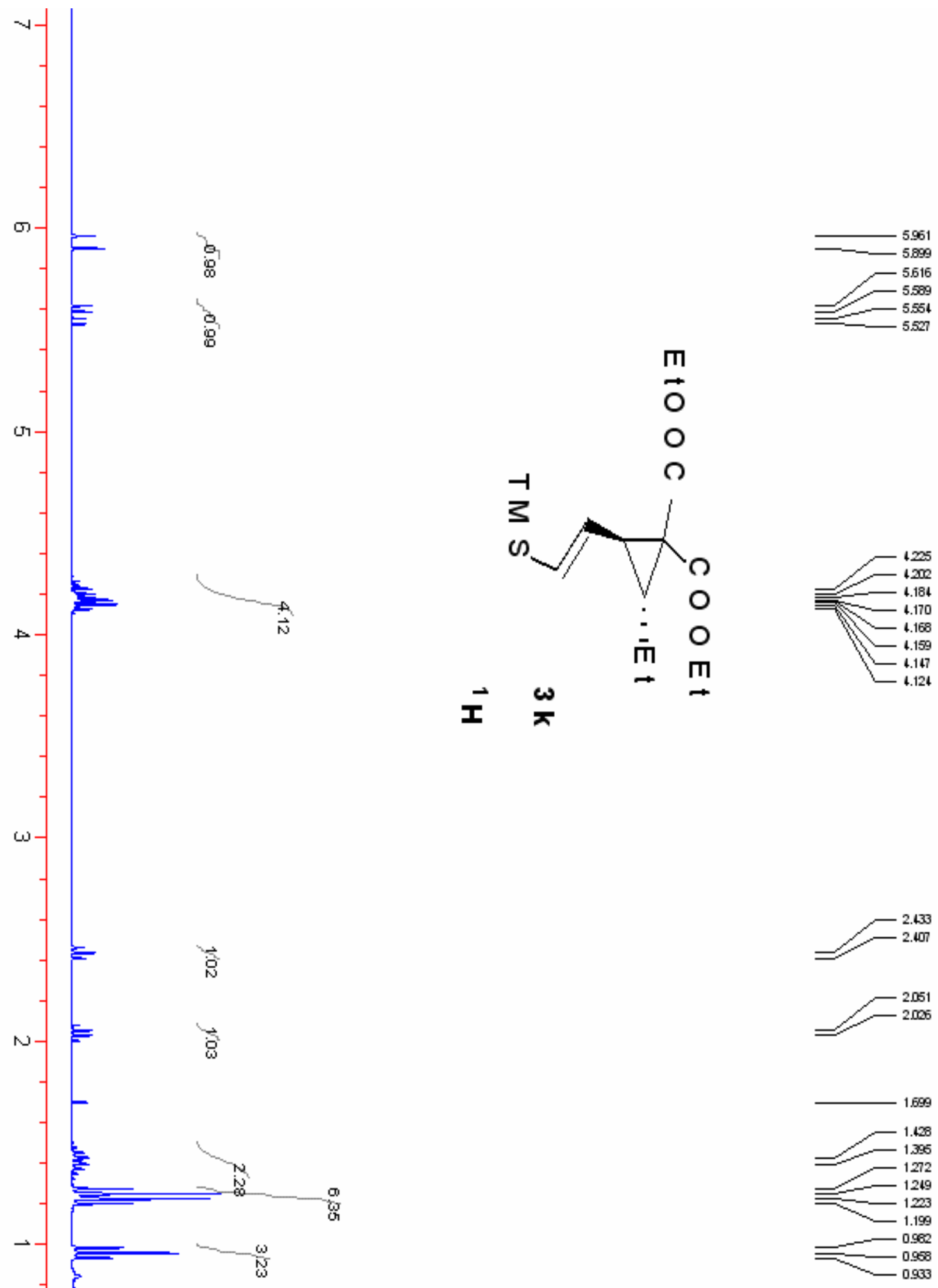

$$
\square
$$

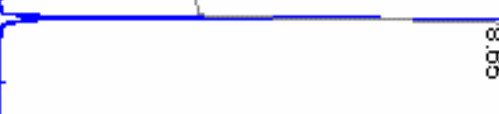

影 


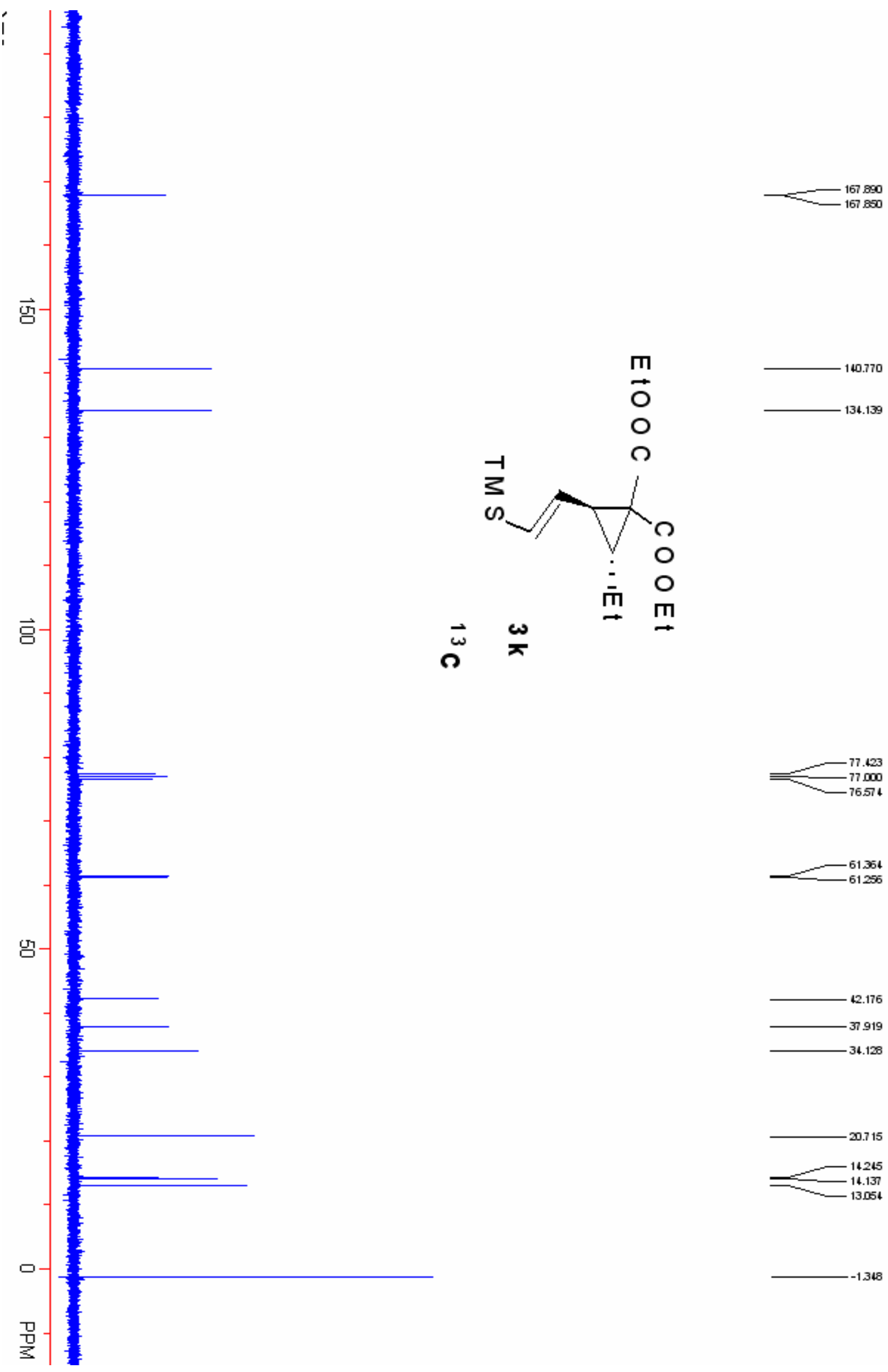



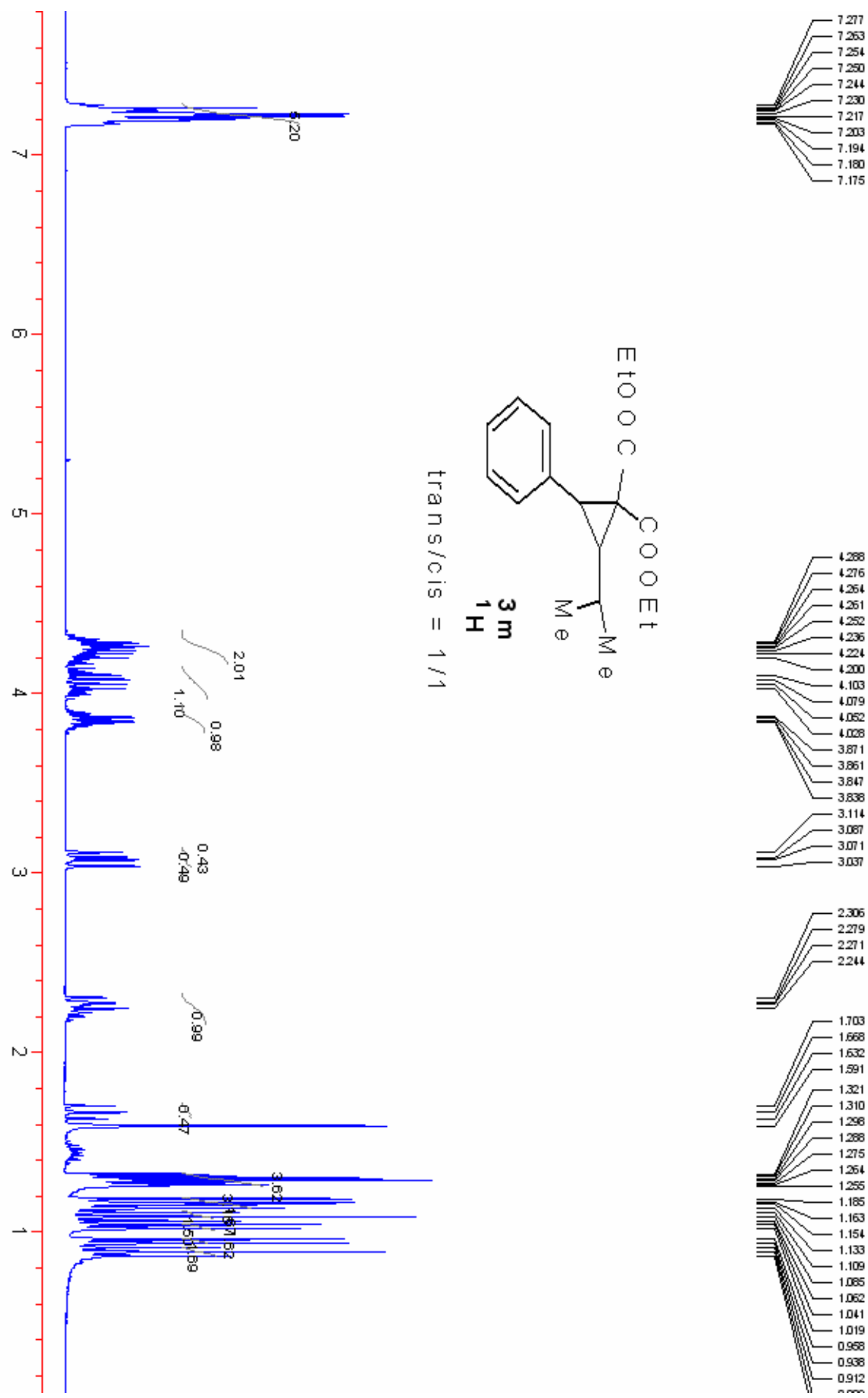


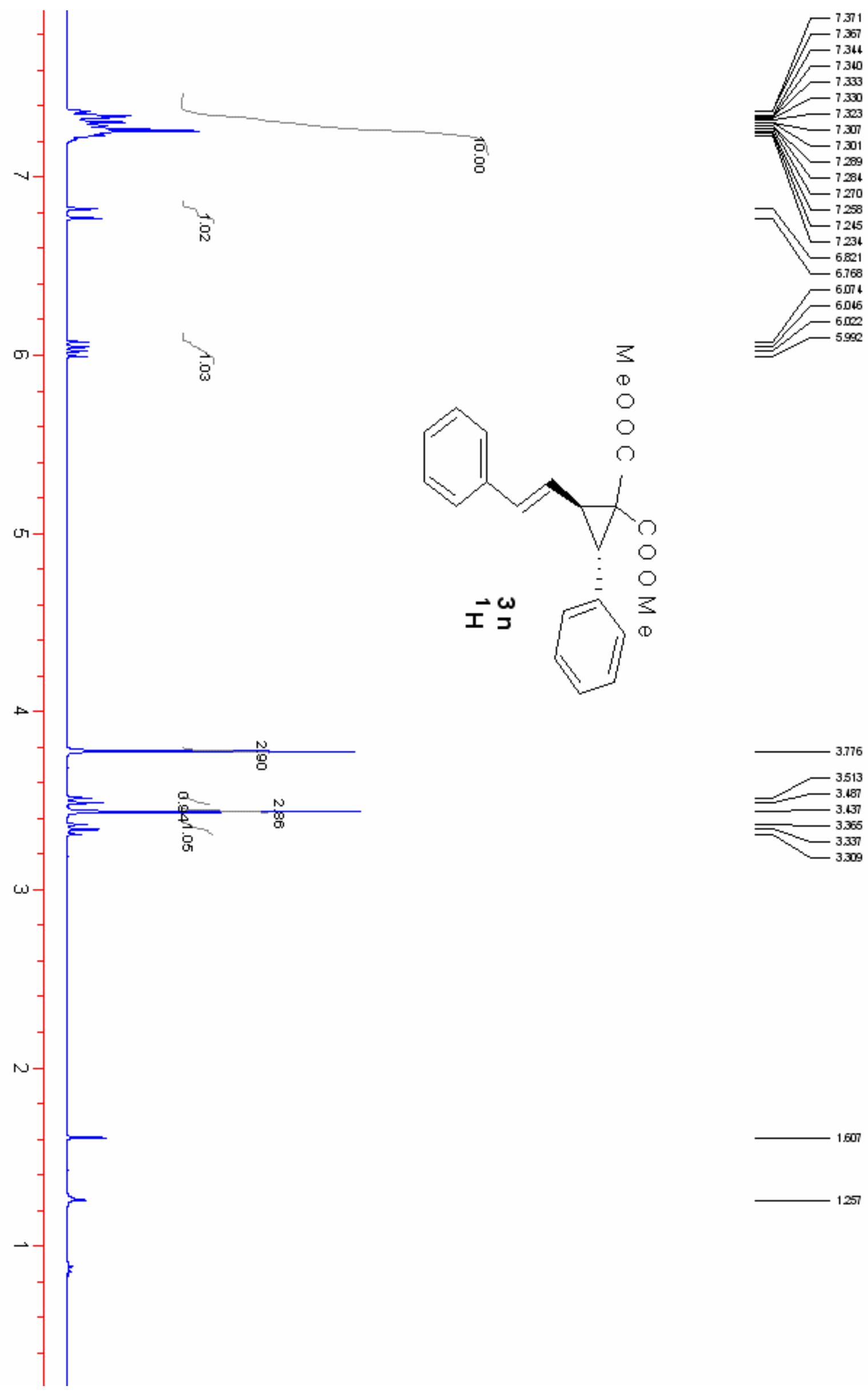




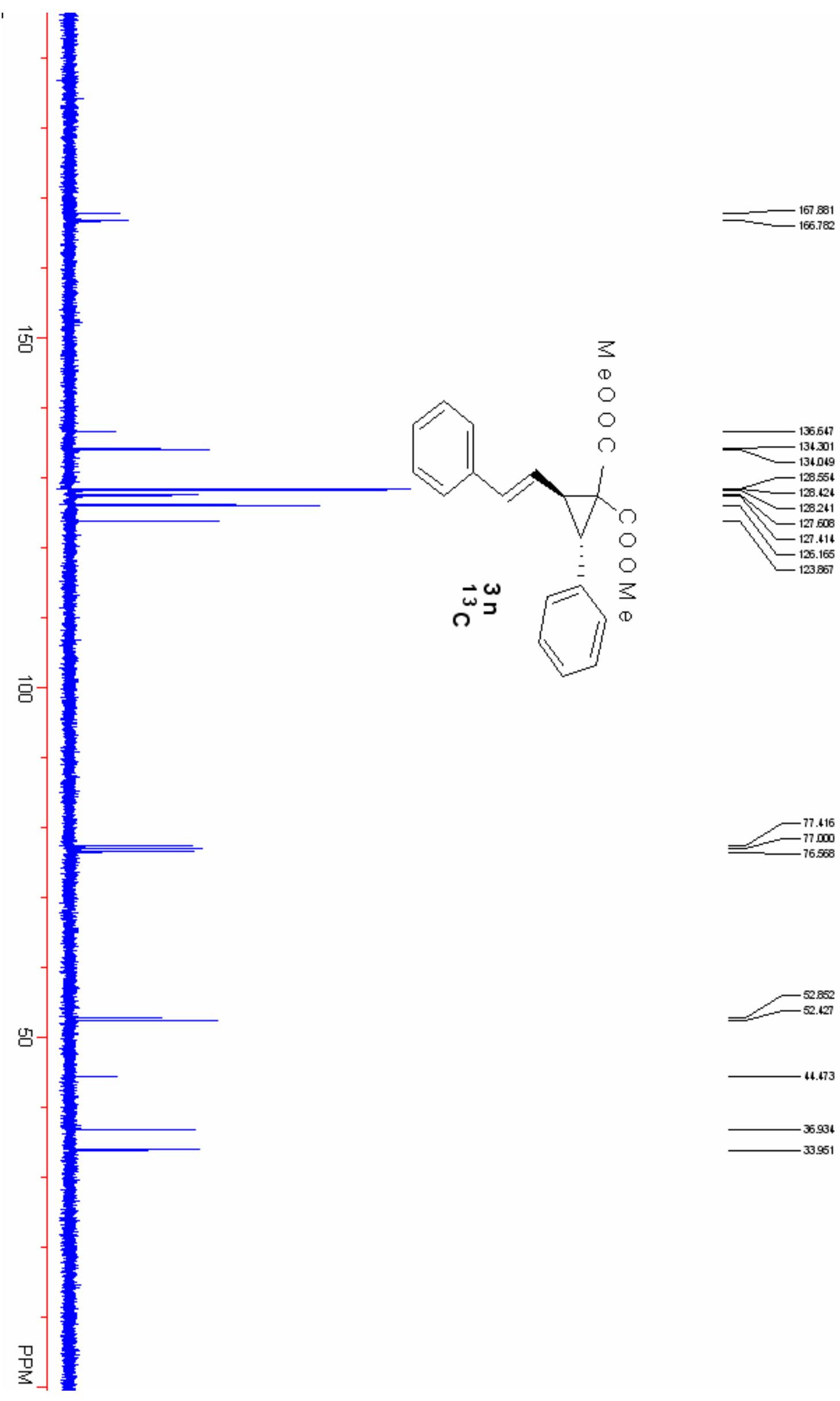



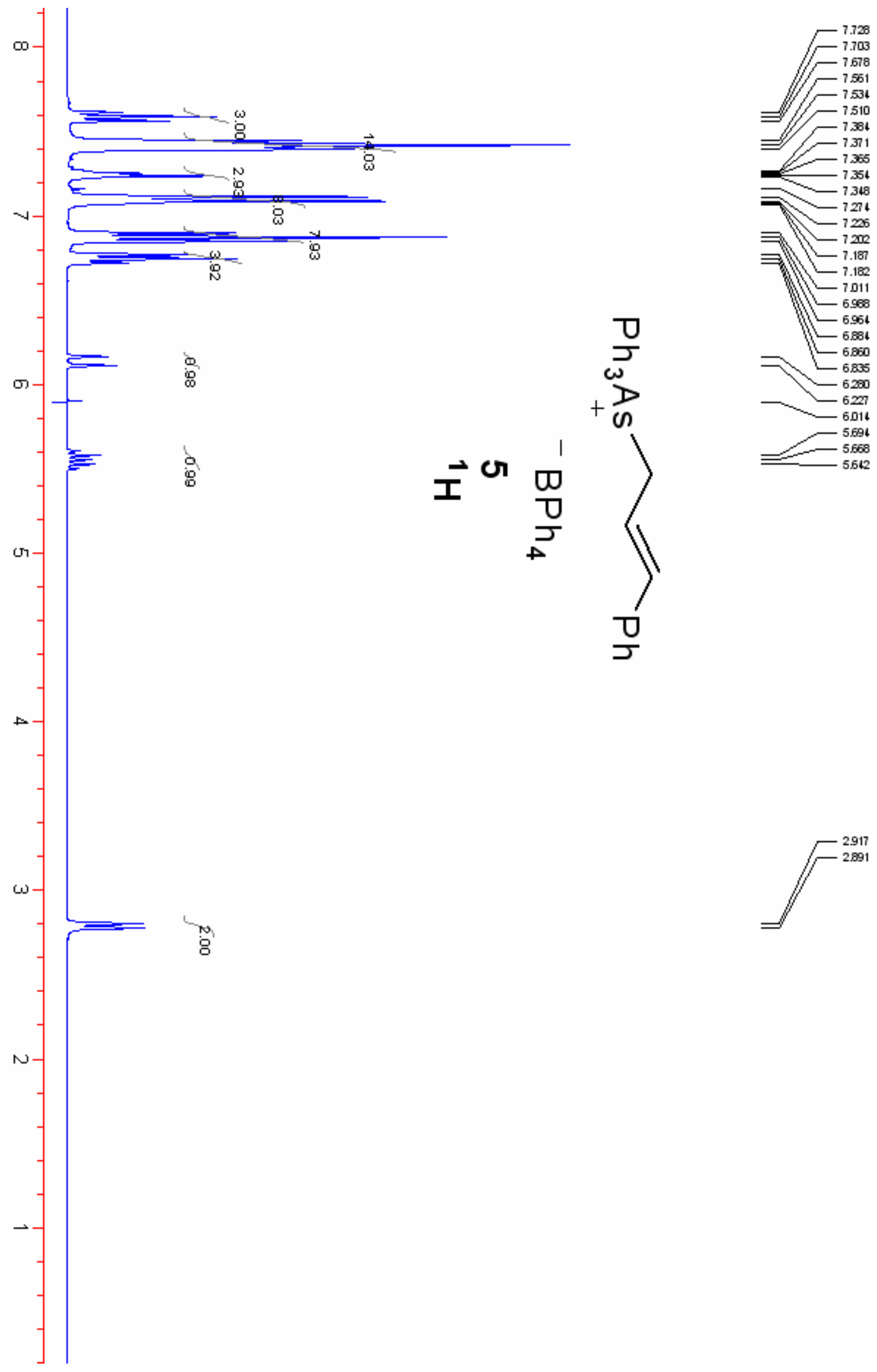

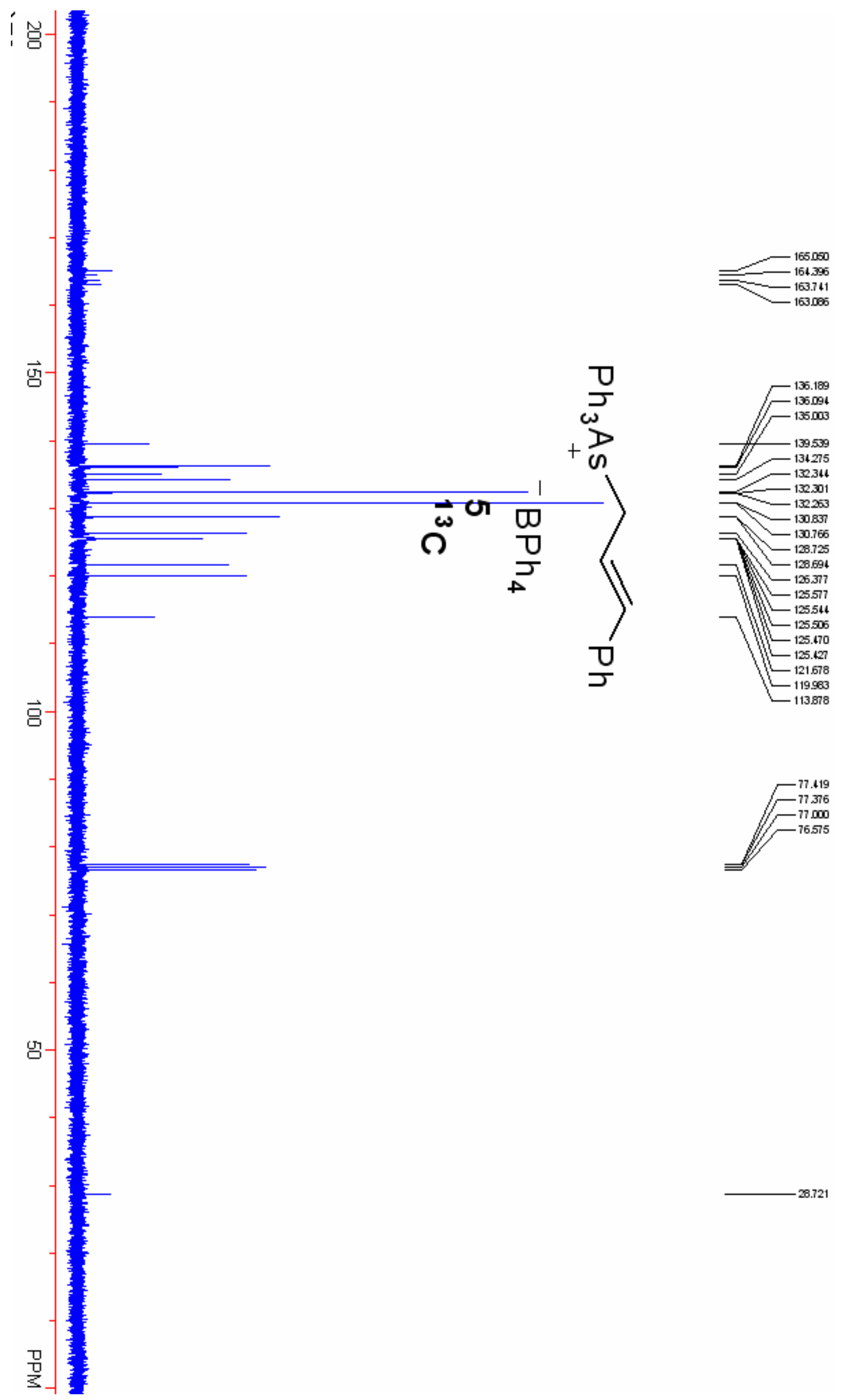


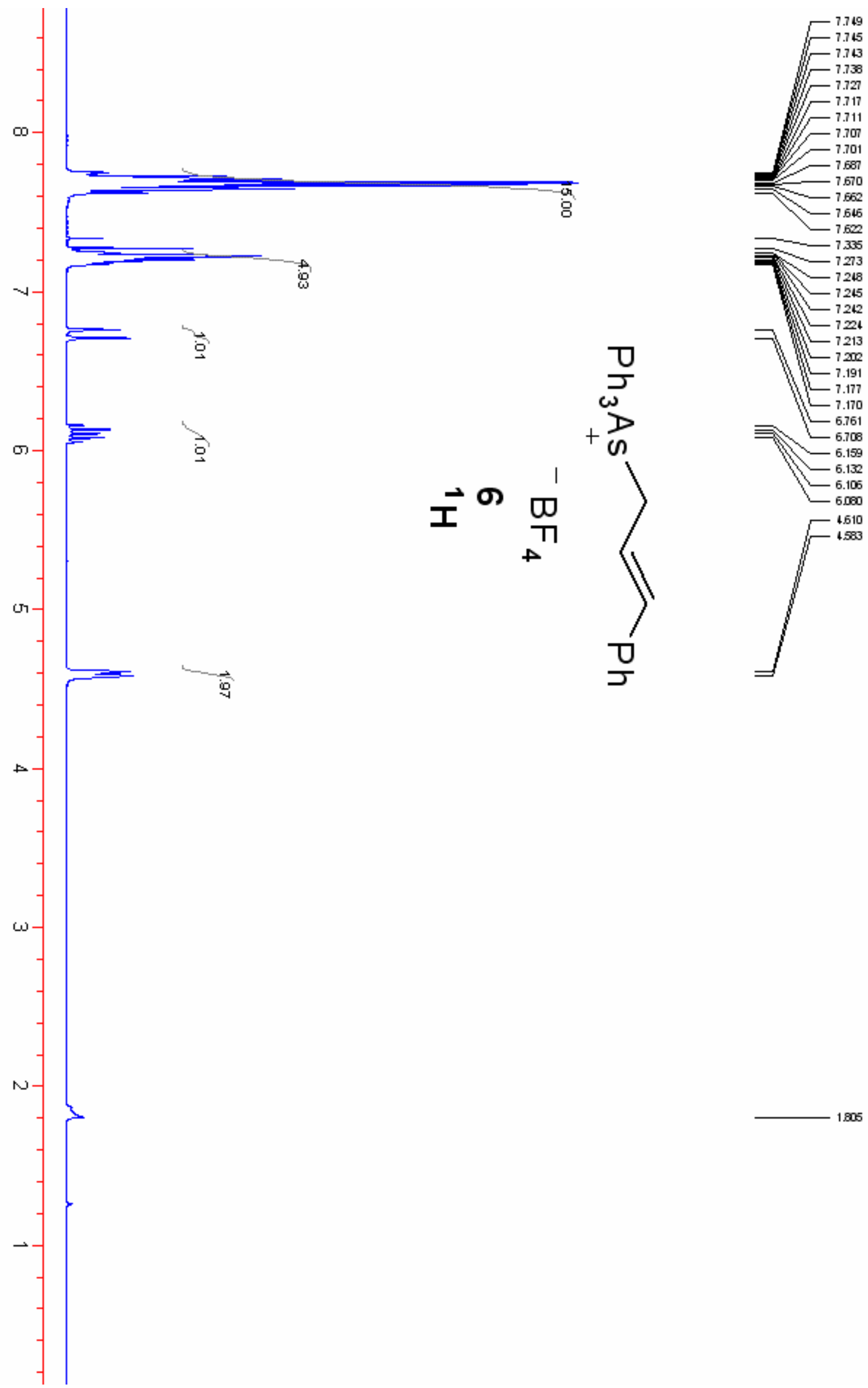




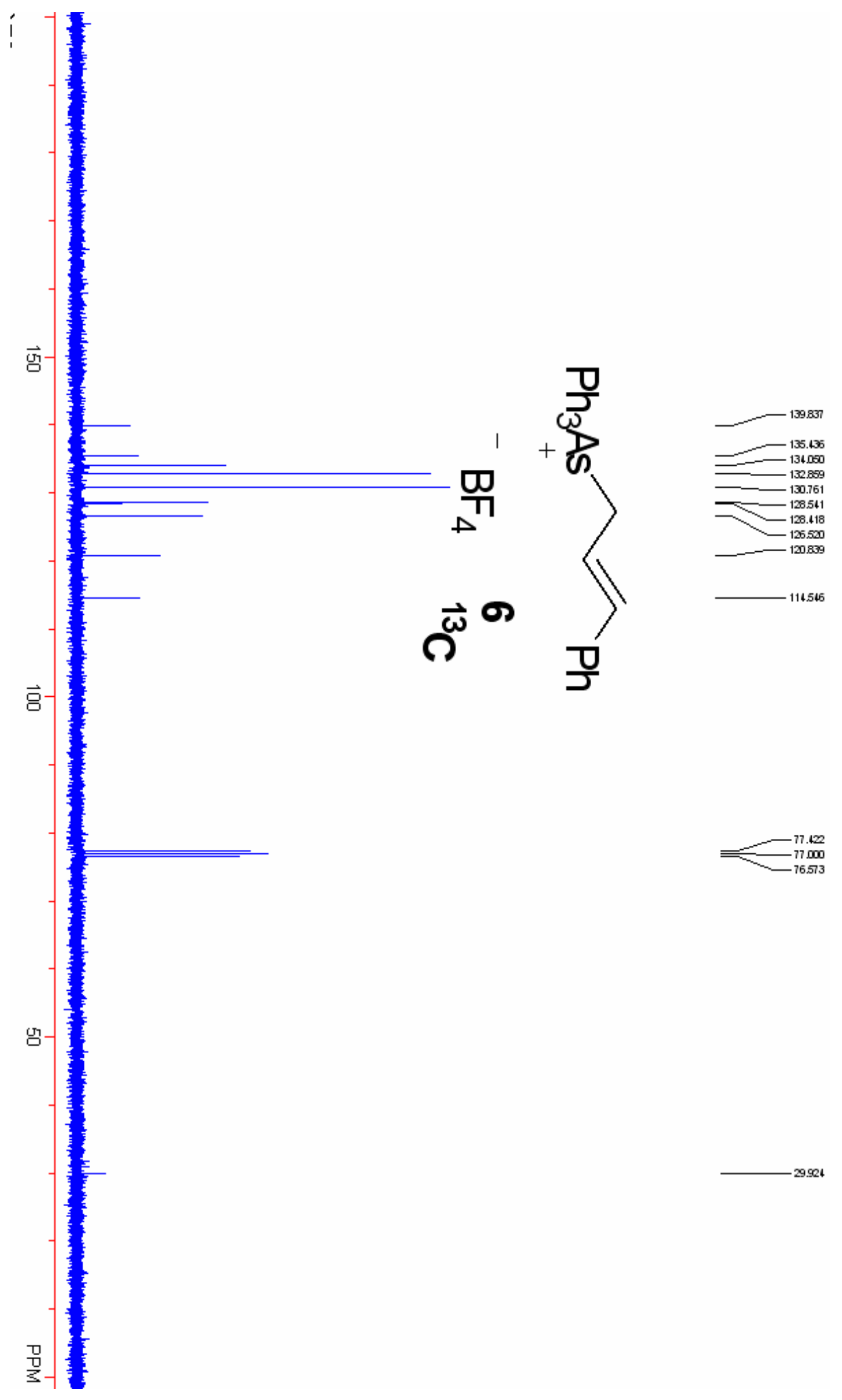

